\title{
The Highly Irregular Firing of Cortical Cells Is Inconsistent with Temporal Integration of Random EPSPs
}

\author{
William R. Softky ${ }^{1,2}$ and Christof Koch ${ }^{2}$ \\ Division of Physics, Mathematics, and Astronomy and ${ }^{2}$ Computation and Neural Systems Program, California Institute of \\ Technology, Pasadena, California 91125
}

\begin{abstract}
How random is the discharge pattern of cortical neurons? We examined recordings from primary visual cortex (V1; Knierim and Van Essen, 1992) and extrastriate cortex (MT; Newsome et al., 1989a) of awake, behaving macaque monkey and compared them to analytical predictions. For nonbursting cells firing at sustained rates up to $300 \mathrm{~Hz}$, we evaluated two indices of firing variability: the ratio of the variance to the mean for the number of action potentials evoked by a constant stimulus, and the rate-normalized coefficient of variation $\left(C_{v}\right)$ of the interspike interval distribution. Firing in virtually all $V_{1}$ and $M T$ neurons was nearly consistent with a completely random process (e.g., $C_{v} \approx 1$ ).

We tried to model this high variability by small, independent, and random EPSPs converging onto a leaky integrateand-fire neuron (Knight, 1972). Both this and related models predicted very low firing variability $\left(C_{v} \ll 1\right)$ for realistic EPSP depolarizations and membrane time constants. We also simulated a biophysically very detailed compartmental model of an anatomically reconstructed and physiologically characterized layer V cat pyramidal cell (Douglas et al., 1991) with passive dendrites and active soma. If independent, excitatory synaptic input fired the model cell at the high rates observed in monkey, the $C_{v}$ and the variability in the number of spikes were both very low, in agreement with the integrate-and-fire models but in strong disagreement with the majority of our monkey data. The simulated cell only produced highly variable firing when Hodgkin-Huxley-like currents ( $I_{\mathrm{Na}}$ and very strong $I_{\mathrm{DR}}$ ) were placed on distal dendrites. Now the simulated neuron acted more as a millisecond-resolution detector of dendritic spike coincidences than as a temporal integrator. We argue that neurons that act as temporal integrators over many synaptic inputs must fire very regularly. Only in the presence of either fast and strong dendritic nonlinearities or strong synchronization among individual synaptic events will the degree of predicted variability approach that of real cortical neurons.
\end{abstract}

\footnotetext{
Received Apr. 30, 1992; revised July 16, 1992; accepted July 23, 1992

We are very grateful to J. Knierim, D. Van Essen, and W. Newsome for their provision of hard-won data for this analysis, to $\mathrm{W}$. Bair for his formatting of it, and to $\ddot{O}$. Bernander for making his simulated neuron available to us. Special thanks are due to $R$. Douglas, F. Crick, and B. Kehoe for many productive discussions and suggestions. We also thank D. H. Perkel, who pioneered these investigations and introduced the parents of one of us (W.R.S.). This research was funded by an NSF Presidential Young Investigator Award, by the Office of Naval Research, by the HFSPO Program in Strasbourg, and by the James S. McDonnell Foundation.

Correspondence should be addressed to W. R. Softky, Division of Biology, 21676, Caltech, Pasadena, CA 91125.

Copyright (C) 1993 Society for Neuroscience $0270-6474 / 93 / 130334-17 \$ 05.00 / 0$
}

[Key words: awake monkey, randomness, coincidence detection, variability, temporal integration, dendritic spikes, integrate-and-fire neuron, visual cortex, biophysical modelingl

When a typical neuron is injected with sufficient current, it fires a rcgular stream of action potentials. But cortical cells in vivo usually fire irregularly in response to a sensory stimulus. What are the cause and function of that irregularity?

Because only the mean firing frequency is reproducible under identical stimulus conditions, while the fine time structure of the irregularities is not, it is widely assumed that information is only carried in the average spike frequency; the fine time structure is usually assumed to be irreproducible "noise." Some electrophysiologists have focused on the idea that the dynamics of the neuronal response may carry significant information (Aertsen et al., 1989; Abeles, 1990; McClurkin et al., 1991). We do not directly address that issue here. Rather, we measure the degree of firing irregularity in cortical cells in the behaving monkey and investigate the possible neuronal sources of the high degree of observed variability.

The irregularity of action potential discharge has been analyzed using the mathematics of stochastic point processes and their intrinsic variability (Perkel et al., 1967; Stein, 1967a,b; Lansky and Smith, 1989; for a recent overview, see Tuckwell, 1989 , and references therein). The firing variability of thalamic and cortical spike trains has been studied experimentally (Poggio and Viernstein, 1964; Noda and Adey, 1970; Burns and Webb, 1976). These and similar studies measured neuronal variability-usually in the form of interspike interval (ISI) distributions - and characterized that variability using various phenomenological statistical distributions (e.g., hyperbolic normal, gamma distribution, etc.). They did not relate the firing variability to the quantitative biophysics of the cells.

One exception is the study by Calvin and Stevens (1968). On the basis of intracellular recordings of cat lumbrosacral motoneurons, they constructed a simple model of the spike generation mechanism. They combined the measured properties of synaptic noise with their model to account for the observed small ISI variability (with an associated coefficient of variability, $C_{\text {I }}$ $\approx 0.05-0.1$ ). They concluded that in the majority of neurons they recorded from, synaptic noise was by itself sufficient to explain the observed variability, without invoking any additional intrinsic noise sources.

Our study uses the same starting point, measuring ISI histograms and their associated coefficients of variation in the case of extracellular recorded units in primary visual cortex (V1) and middle temporal visual area (area V5 or MT) of the awake behaving monkey. Unlike the lumbrosacral motoneurons, the 
neocortical units have a very high degree of irregularity, with $C_{1}$ ranging between 0.5 and 1.0 . We attempt to understand the origin of these values by two different theoretical methods: modiffed integrate-and-fire models, and simulations of detailed compartmental models of cortical pyramidal cells. Our analysis reveals a strong contradiction between the large observed interspike variability at high firing rates and the much smaller values predicted by well-accepted analytical and biophysical single-cell models. This contradiction does not exist for high variability at low firing rates, which is consistent with the models of Wilbur and Rinzel (1983) and Bugmann (1990).

The manuscript is organized in four parts: data, analytical models, compartmental models, and discussion. A brief report of this work has appeared previously (Softky and Koch, 1992).

\section{Electrophysiological Data}

We used data from two different laboratories. In both cases, extracellular spike trains were recorded from cells in visual cortex of awake adult macaques. Our primary interest was neither in the nature of the stimuli used nor in the cells' selective responses to these stimuli, but only in the statistical properties of neuronal firing.

The first set of data ("Vl" data) came from an investigation of the influence of the nonclassical receptive field (RF) on singleunit activity in the primary visual cortex of two alert and behaving macaque monkeys (Macaca fascicularis; Knierim and Van Essen, 1992). Data were only accepted for trials during which the monkey fixated or performed a fixation-related task. The cells were stimulated by a variety of flashed bars of various orientation in the center of the classical RF, and in some cases additionally stimulated by either parallel or perpendicular oriented bars outside the classical RF (Knierim and Van Essen, 1992). We used 1184 single, well-isolated spike trains of $1 \mathrm{sec}$ duration recorded from 16 cells at a temporal resolution of 1 msec. Only one of these cells showed any bursting activity (as defincd bclow), and was rejected.

The second set of data (referred to in the following simply as "MT" data) was recorded during an investigation into the relationship between motion discrimination and the behavior of single units in area MT (or V5), a region of extrastriate visual cortex concerned with motion processing $(M$. mulatta; Newsome et al., 1989b; Britten et al., 1992). In brief, three monkeys were trained to report the direction of motion of a random dot display in which a fixed fraction of dots (the amount of "motion coherency") moved coherently in one direction while the remainder moved randomly in all directions (Newsome and Pare, 1988). The amount of motion coherency, as well as the direction of motion, was varied across trials. During a single trial, the monkeys, whose heads were restrained, had to fixate a cross. If fixation was broken - as monitored by a search coil-the trial was terminated.

Standard electrophysiological procedures were used to identify and record single MT units in three alert and behaving monkeys (Mikami et al., 1986). The two-threshold window discriminator produced pulses corresponding to single action potentials whose time of arrival was recorded with $1 \mathrm{msec}$ resolution. Care was taken to record only single-unit activity. Altogether, the activity from 409 neurons was recorded, each trial usually being 2 sec long. Figure 1 shows a sample spike train, poststimulus time histogram (PSTH), and ISI histogram from a typical MT recording.

For our analysis, we used a subset of these trials. We rejected

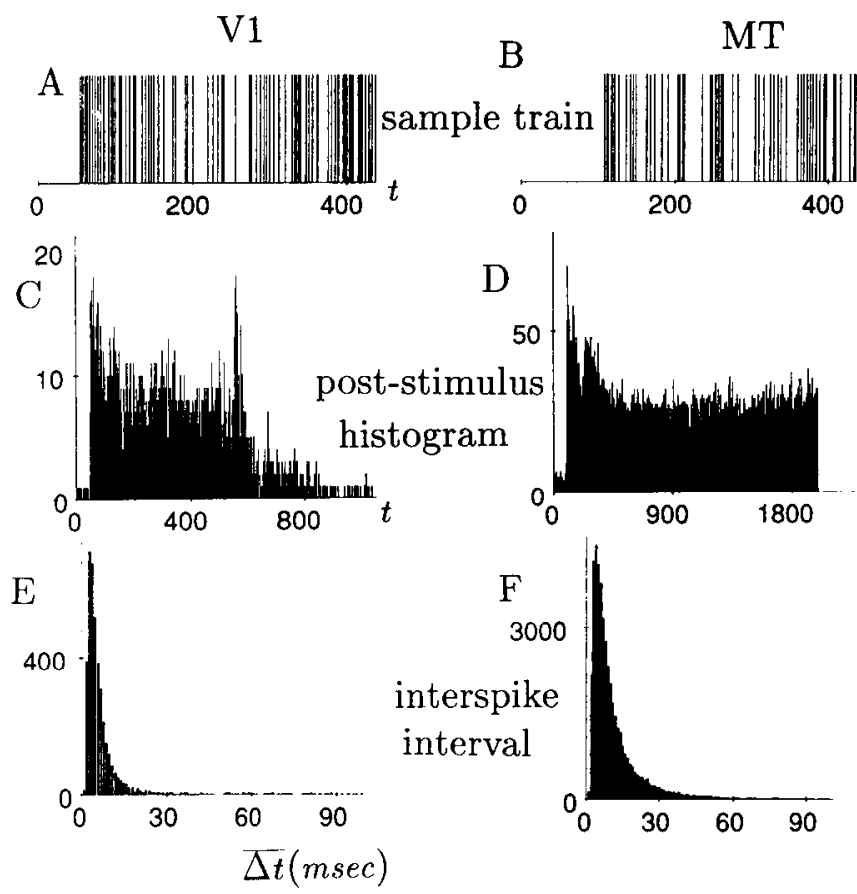

Figure 1. Firing statistics of neurons in areas VI and MT. $A$ and $R$, Sample spike trains from one of the fastest-firing nonbursting neurons recorded in each area. $C$ and $D$. PSTHs from the same neuron. $E$ and $F$, ISI histograms from the same neuron. These neurons are "typical" in that their firing times seem nearly random at all observed firing rates.

all spike trains that contained any dominant ISIs characteristic of "bursting" behavior. A "bursting" neuron frequently fires a pair of action potentials within a short time $(<1-3 \mathrm{msec})$, a situation characterized by a sharp peak in that range on the ISI histogram. More specifically, we rejected any neuron whose ISI histogram (sce below) containcd more than twice as many counts in the $2 \mathrm{msec}$ bin as in the $5 \mathrm{msec}$ bin. These criteria yielded a subset of 233 nonbursting neurons.

In general, we did not find any significant difference between the degree of variability of V 1 or MT neurons. Therefore, except when otherwise explicitly noted, we will lump these two sets of experimental data together.

\section{Analysis Method}

\section{Parameters and normalization procedure}

The spikes following the stimulus onset arrived at times $\left\{t_{i}\right\}$. Thus, the interspike interval (ISI) is

$$
\Delta t_{i}=t_{i+1}-t_{i} .
$$

We will analyze histograms of these ISIs through two of their parameters. One is the mean of the histogram (the average interspike time $\overline{\Delta t}$ ):

$$
\overline{\Delta t}=\frac{1}{S_{j}-1} \sum_{i=1}^{S_{i}} \Delta t_{i}
$$

where $S$, is the number of spikes in the train. The other parameter is the standard deviation about that mean, which is

$$
\sigma_{د t}=\sqrt{\frac{1}{S_{j}-1} \sum_{i=1}^{S_{j}-1}\left(\Delta t_{i} \cdot \overline{\Delta t}\right)^{\prime}} .
$$




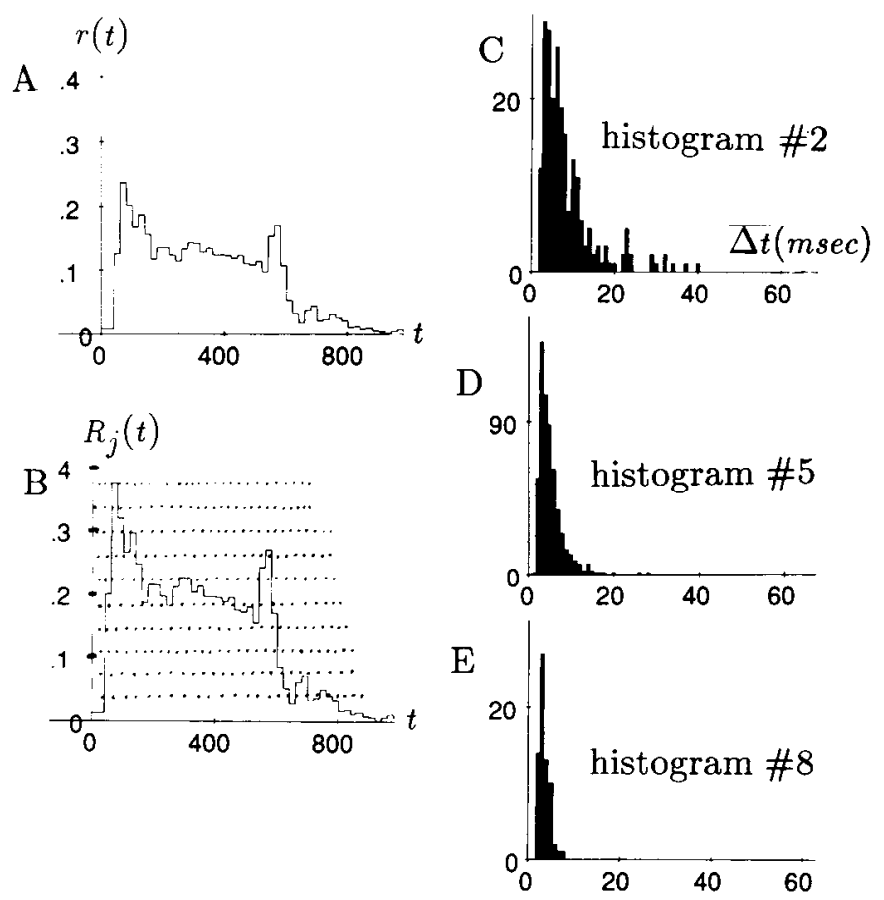

Figure 2. Firing variability analyzed by multiple ISI histograms. The firing rate of a cell depended on both stimulus efficacy and poststimulus time. ISI histograms were made from such data by segregating ISIs according to the approximate instantaneous rate $r(t)$, according to the following steps. $A, r(t)(\mathrm{Hz})$ was calculated for any particular cell from the PSTH for all the responses of that cell to stimulation. $B$, The instantaneous rate $R_{j}(t)$ for train $j$ was computed by multiplying $r(t)$ by the ratio of the total number of spikes $S_{j}$ of that train to the cell's average number of spikes $S_{\text {avg. }}$. Here, $S_{\text {avg }}=83$ and $S_{j}=131$. Each ISI was placed into 1 of 10 ISI histograms, so that each histogram represented a roughly constant firing rate: histogram 0 was slowest, and histogram 9 was fastest. $C-E$, Three of the 10 histograms for the V1 cell of Figure 1 are shown here (spikes between $50 \mathrm{msec}$ and $100 \mathrm{msec}$ are not shown here, but were included in our analysis.) These ISI distributions are typical of cortical cells described elsewhere: a virtual absence of ISIs below 2 msec indicates the refractory period and the absence of "bursting" behavior, and the distribution is very wide relative to its mean. Each histogram's shape parameter $C_{V}$. contributed one point in Figures 3, 9, and 13 .

These two values together yield a measure of the variability of the spike train, the dimensionless coefficient of variation, which describes the relative width of the ISI histogram:

$$
C_{1}=\sigma_{\Delta t} / \overline{\Delta t} \text {. }
$$

For a very regular spike train ("pacemaker"), the ISI histogram will have a very narrow peak and $C_{r} \rightarrow 0$. In the case of a random spike train (a Poisson process or shot noise), the $\Delta t_{i}$ are exponentially distributed and $C_{i}=1$. The coefficient of variation can be larger than one in the case of a multistate neuron (Wilbur and Rinzcl, 1983).

This analysis could not be applied directly to our data, because both V1 and MT neurons "adapted," in that their firing rates decreased to roughly half the initial value during the first 100 $300 \mathrm{msec}$, despite a constant visual stimulus. Moreover, because more than one stimulus was used on each cell, the number of spikes varied significantly between trains. We found that the relative contributions of transient and sustained firing varied only slightly for different stimuli, because the poststimulus time histograms (PSTHs) for different stimuli all had approximately the same shape.

Because such nonstationary (variable-moment) statistics are difficult to analyze, the goal of the analysis was to arrive at an approximate estimate of $C_{V}$ at a near-constant output rate without artificially broadening the ISI histograms due to the change in mean firing rate. That is, we attempted to eliminate the artificial source of variance induced by adapting rates by separating spikes into many histograms, each representing a roughly constant firing rate. Our method was to compute the approximate instantaneous firing rate $R$. We then used $R$ to segregate spikes into 10 different histograms. The highest $R$ (early times with strong stimuli) binned corresponding spikes in the "fastest" histogram, the lowest $R$ (the tail end of the weaker stimuli) put spikes into the "slowest" histogram, and intermediate $R$ stored spikes in corresponding histograms in between. The predicted values of $R$ and its resulting range were calculated separately for each cell, as follows.

The major simplifying assumption was that the cell's instantaneous rate at time $t$ during any particular experimental trial $j$ depended only on the total number spikes $S_{j}$ in that train and on the cell's average instantaneous response $r(t)$, averaged over all $m$ experimental trials for that particular cell. The instantaneous response $r(t)$ was taken directly from the PSTH of the cell for all $m$ stimuli, coarse-graining $t$ to bins $20 \mathrm{msec}$ wide (indexed by $\tilde{t}=0,20,40 \mathrm{msec}$ ). Thus, if $S_{j}(\tilde{t})$ is the number of spikes in train $j$ falling in bin $i$, then

$$
r(t)=r(\tilde{t})=\frac{1}{20 m} \sum_{j=1}^{m} S_{j}(\tilde{t})
$$

The true instantaneous rate $R_{f}(t)$ is then assumed to be the product of $r(t)$ and $S_{j}$, normalized by $S_{\text {avg }}$, the average number of spikes in a train for that cell [i.e., $S_{\text {ave }}=(1 / m) \sum_{j=1}^{m} S_{j}$ ]:

$$
R_{j}(t)=S_{S_{\mathrm{avg}}} \times r(t)
$$

The $S_{i}$ term in Equation 6 represents the efficiency of the stimulus, that is, how many spikes the cell fired over the entire recording interval (e.g., $2 \mathrm{sec}$ in the case of the MT recordings) in response to a particular visual stimulus, while $r(t)$ describes the time course of neuronal adaptation over all stimuli used for that particular cell. Note that only the parameter $S$, was used in the Newsome et al. (1989a) study-for which the MT data analyzed here were generated - for the evaluation of neuronal thresholds.

Each ISI in any spike train for a particular cell was then placed into 1 of 10 different histograms according to its associated $R_{i}$ value. The maximum rate $R_{\max }$ (defined over all stimuli conditions for that cell) was used to define 10 equally spaced rate intervals from 0 to $R_{\max } \mathrm{Hz}$, that is, (0-0.1) $R_{\max },(0.1-0.2) R_{\max }$, $(0.9-1.0) R_{\max }$. Each of these ISI histograms had a temporal resolution of $1 \mathrm{msec}$ and a total range of $100 \mathrm{msec}$ (longer ISIs were not necessary for this analysis of high firing rales). For each spike train $j$, both $\Delta t_{t}$ and $R_{f}\left(t_{i}\right)$ were computed from the original data, following Equations 1 and 6 . Then, $\Delta t$, was assigned to the time $t$ at the center of its ISI [i.e., $t=\left(t_{i}+t_{i+1}\right)$ / 2). Finally, $\Delta t_{i}$ contributed one count to the appropriate ISI bin in the particular histogram whose rate range included $R_{i}(t)$. Figure 2 illustrates this procedure for a "typical" V1 cell. Here, $R_{\max }=380 \mathrm{~Hz}$ and $S_{\text {avg }}=83$ spikes in $1 \sec (83 \mathrm{~Hz})$. The average time course $r(t)$ and $R_{(}(t)$ (for the fastest-firing train) are shown in Figure $2, A$ and $B$. Three of the associated 10 histograms, into which a total of 4009 ISI values were placed, are also illustrated. Note that the instantaneous rate $R_{f}(t)$ was only used 
to determine into which histogram any particular value of $\Delta t_{i}$ should be placed.

Because the histograms with highest $R$ only contained the earliest spikes of the few fastest trains, they typically had far fewer spikes than the intermediate histograms (see Fig. 2). Nonetheless, these fast histograms usually contained enough spikes to be statistically significant, judging by the error bars in $C_{V}$ as calculated below. In addition, the fastest histograms had mean rates typically twice as fast as the cell's average (adapted) response to a strong stimulus over $1-2 \mathrm{sec}$.

The parameters $\overline{\Delta t}, \sigma_{\Delta l}$, and $C_{l}$, were then calculated from each of the 10 histograms [without using $R(t)$ ]. Error bars were derived from the counts in individual histogram bins by treating those bin counts as Gaussian random variables. For example, if $M_{د t}$ counts fall in a single bin $\Delta t$, then we assume the uncertainty in $M_{د t}$ is $\sigma_{M}=\sqrt{M_{\lambda}}$, and we propogate errors as random variables to get

$$
\overline{\Delta t}=\frac{1}{100} \sum_{\Delta t=0}^{s^{\prime}}\left(M_{\Delta t} \pm \sqrt{M_{\Delta t}}\right) \Delta t
$$

and

$$
\sigma_{\Delta i}^{2}=\frac{1}{100} \sum_{s t=0}^{s_{t=100}}\left(M_{s_{i}} \pm \sqrt{M_{\Delta}}\right)(\Delta t-\overline{\Delta t})^{2}
$$

The resulting $C_{1}$, values were plotted against $\overline{\Delta t}$ (Fig. 3) for all but the slowest two histograms for each cell (i.e, for all histograms within which the instantaneous rate varied by no more than $33 \%$ ). $C_{1}$. values from histograms with less than 10 counts were also excluded, so that each cell contributed eight or fewer points to a plot of $C_{l}$. This entire normalization procedure was repeated for each of our 249 cells. Had $C_{V}$, been calculated only from the total ISI histogram for a single cell (i.e., without using the multihistogram method), equally high values would have resulted $\left(C_{1} \approx 0.7-1.1\right)$, but such histograms would have confounded high and low firing rates and would thus have been difficult to interpret.

\section{Inaccuracy of analysis method}

The statistics of spike trains are not precisely defined for nonstationary processes. But we are only concerned with the approximate variability of the spike train, so let us suppose for the moment that our data represent a simplified process in which each ISI was generated randomly, according to some distribution with fixed $C_{1}$ and variable rate. Would the analysis method described above reveal the true (generating) value of $C_{r}$ ? We will discuss some of the limitations of this multihistogram analysis method, and then show a simulation that suggests that our method is indeed suited to our purposes.

The above method underestimated $C_{1}$, for low firing rates, because some long ISIs were excluded from their proper histograms. Some spike trains were only $500 \mathrm{msec}$ long; thus, ISIs longer than that duration obviously could not be counted. A more stringent limit was the width of the ISI histogram from which $C_{1}$. was calculated (100 bins of $1 \mathrm{msec}$ each), which truncated the tails of ISI distributions with large $\overline{\Delta t}$ and high $C_{F}$. (e.g., $\overline{\Delta t} \geq 25 \mathrm{msec}$ ). In all these cases, truncating the tail of a broad ISI distribution artificially narrows the histogram, and reduces the estimated $C_{1}$, below its true value.

In other cases, this analysis overestimated $C_{1}$. This artificial broadening of the ISI histogram can occur, for instance, when the firing rate changes during the rate-averaging period $\tilde{t}$ : a smooth variation in firing rate would be misconstrued as a high

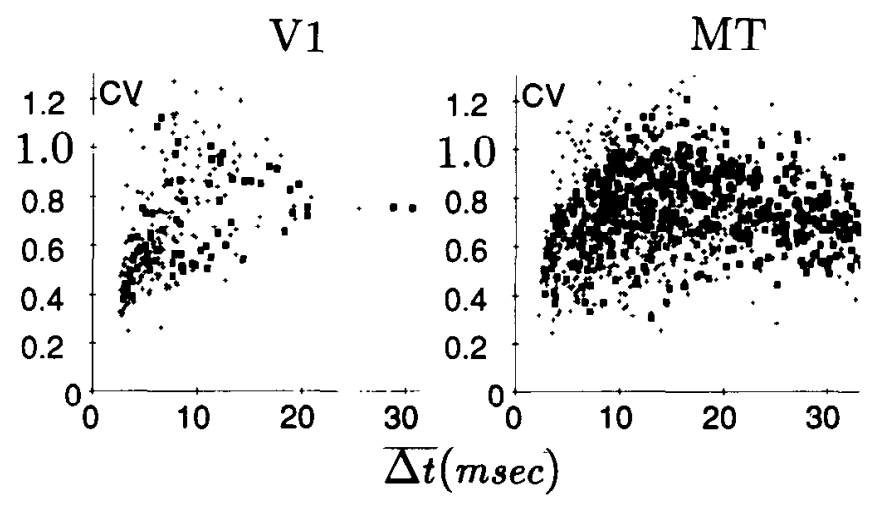

Figure 3. Variability of neurons in areas V1 and MT. $C_{1}$. characterizes the normalized width of a histogram. The scattered points were obtained from ISI histograms like those in Figure 2 (only points with $\overline{\Delta t} \leq 30$ msec are shown). Squares are reliable points $\left(\sigma_{c_{2}} / C_{1} \leq 0.1\right)$; pluses are less reliable $C_{1}$ values. The main systematic bias of the analysis method was to underestimate $C_{1}$ for large ISIs $(\overline{\Delta t} \geq 20 \mathrm{msec})$. The slightly higher firing rates of the V1 neurons resulted from the choice of such faster neurons for analysis; no other differences are apparent between the two areas.

random variability. Although this effect obviously occurs during the onset of spike adaptation (in the early part of the PSTH, when the average rate changes most quickly), it can also occur at the lowest rates measured for one cell, for which a single histogram has a higher fractional variability than at higher rates (e.g., a $60-90 \mathrm{~Hz}$ histogram contains $33 \%$ frequency variability, vs. $10 \%$ for a $270-300 \mathrm{~Hz}$ histogram).

A further artifact occurred at high firing rates, when the width of a single time bin ( $1 \mathrm{msec}$ ) becomes comparable to the shortest ISIs observed ( $\overline{\Delta t} \approx 2 \mathrm{msec}$ ). This effect is most pronounced when the true histogram is very narrow and steep sided, so that the "rounding error" (about $0.5 \mathrm{msec}$ ) induced by shifting each ISI to a neighboring bin changes the histogram's width significantly.

In order to quantify these combined effects, we numerically simulated spike trains with the following characteristics: (1) each ISI was generated by a gamma probability distribution with constant and known $C_{1}$, variable mean rate, and a resolution of $1 \mathrm{msec}$; (2) each train was $500 \mathrm{msec}$ long; (3) the mean rate dropped linearly to 0.33 of its starting value within $250 \mathrm{msec}$ (modeling adaptation); (4) starting rates for different trains were chosen to give a range of $\overline{\Delta t} \approx 2-30 \mathrm{msec}$ (comparable to the monkey ISIs) between the very fastest and very slowest mean ISIs observed. The more variable of these artificial trains looked just like real trains from monkey. At each $C_{1}, 500$ simulated trains at different rates were analyzed together by our normalization method described above. In addition, the slowest 100 trains were separately analyzed, to resolve better the slowest ISIs.

The comparison of the $C_{1}$ values yielded by this analysis with the $C_{V}$ of the random processes generating the trains (Fig. 4) confirms the two points outlined above: this method systematically overestimates $C_{1}$ when both $C_{1}$ and $\overline{\Delta t}$ are low, and systematically underestimates $C_{1}$, when both are high. But for fast-firing, highly variable cells - like those observed in our analysis - this method introduces a systematic bias that is no greater than a few percent.

While our normalization method seems to account for spurious effects introduced by a variable firing rate, there remains the fact - not modeled by the foregoing simulation-that $C_{1}$. itself can vary as well. In fact, this changing $C_{1}$ is observed in 


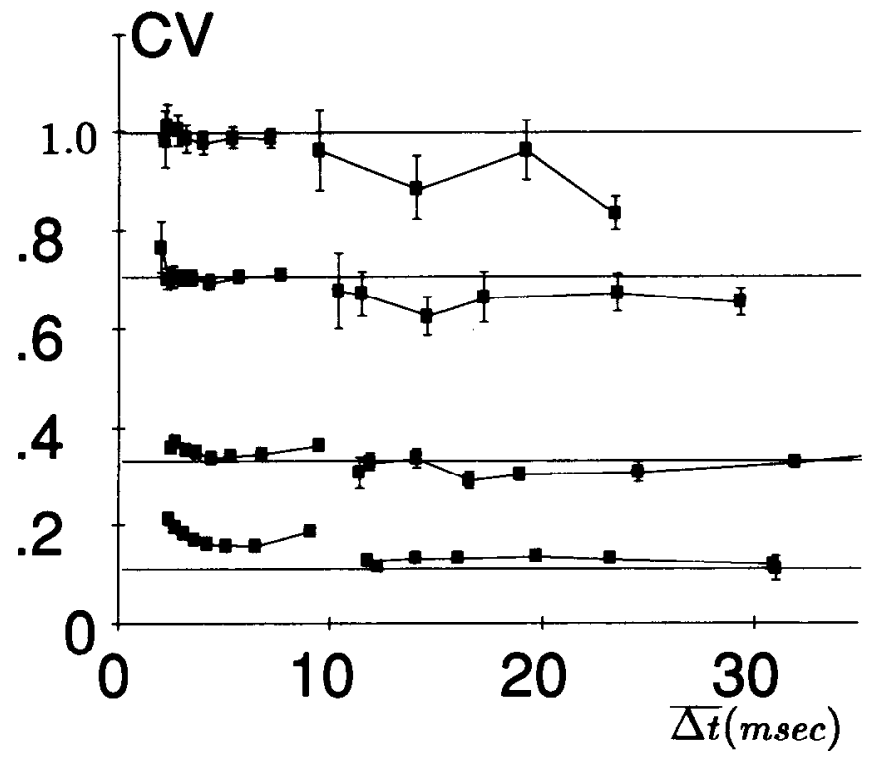

Figure 4. Accuracy of the multihistogram normalization method. Computer-generated spike trains (having roughly the same mean firing rates and adaptation course as the monkey data) were randomly generated from gamma function ISI distributions of various $C_{v}(1.0,0.71$, $0.33,0.11$ ) with $1 \mathrm{msec}$ resolution. We analyzed these fake trains by the same method used for the monkey data, analyzing slow and fast trains separately to resolve $C_{v}$ at both long and short ISI values. The resulting $C_{V}$ values (connected squares) were compared with the rateindependent $C_{V}$ value of the generating distribution (horizontal lines). The analysis method underestimated high $C_{V}$ at long ISI values, and overestimated low $C_{V}$ at short ISI values. But high $C_{V}$ values at short ISI (like those observed in monkey) were not systematically biased more than a few percent. Thus, the drop in $C_{V}$ at the left of Figurc 3 is rcal, but the drop at the right is an artifact of the analysis method.

the monkey cells: lower firing rates of individual cells have a higher $C_{V}$ (see Refractory Period and Adaptation sections).

If several processes with the samc rate (and thus the same $\overline{\Delta t}$ ) but different $C_{V}$ values have their ISIs binned in the same histogram, the resulting histogram (e.g., a sharp peak on a broad base) will have the same mean $\overline{\Delta t}$ as each process separately. The new value of the variance about that mean is given by the weighted mean of the two variances of the individual histograms, so that the composite $C_{V}$ value will be bounded by the $C_{V}$ values of those separate processes. Because our claim in this article is that the $C_{V}$ values we observe in monkey lie outside a certain predicted range, the fact that those observed $C_{V}$, values may themselves only be averages of several true values still requires that most of the true values remain outside the range.

As a further check that our high $C_{V}$ values did not result from peculiarities of the normalization method, we compared ratenormalized values with those obtained from adapted, constantrate portions of MT spike trains without time-dependent normalization: the two methods gave identical $C_{V_{v}}$ values. We also found that changing the PSTH bin size from $\tilde{t}=20 \mathrm{msec}$ to $\tilde{t}$ $=5 \mathrm{msec}$ made no difference in the computed $C_{V}$, even during strong adaptation.

\section{Variability in the interspike interval}

The approximate $C_{V}$ values measured and illustrated in Figure 3 are in good agreement with reports of $C_{V}$ at lower firing rates of cortical cells (Noda and Adey, 1970; Burns and Webb, 1976): $C_{V} \approx 0.5-1$. Visual inspection of the $C_{v}$ plots did not reveal any

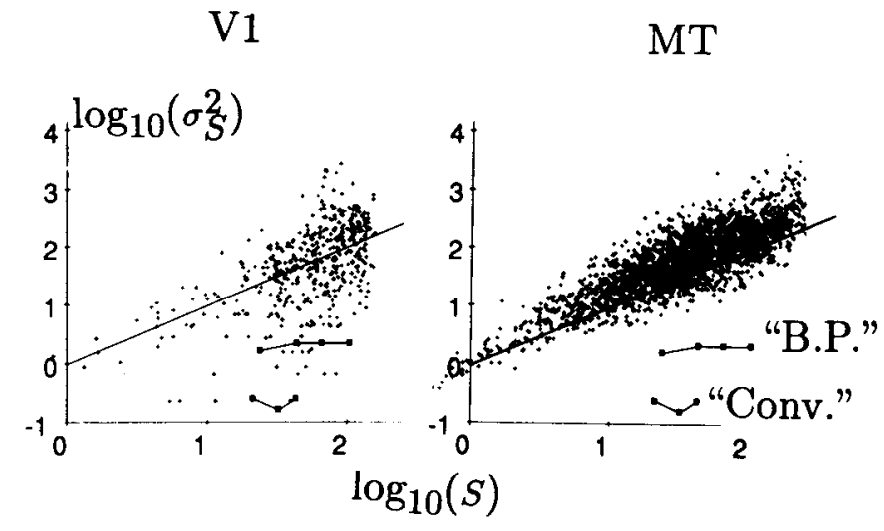

Figure 5. Comparison of the variance in spike count for monkey and simulated pyramidal cells. Plots of the mean number of spikes $S$ in a train for a continuous stimulus and the variance $\sigma_{S}^{2}$ about that number indicate the firing variability over longer times; the log-log scale contains values from a few spikes to hundreds. Values for monkey cells are pluses, in agreement with those obtained for the same areas by Snowden et al. (1992). The diagonal line represents the prediction for a purely random Poisson process at constant rate $\left(\sigma_{S}^{2}=S\right)$. The connected squares on both graphs are values given by the b.p. and c. simulations, and have far lower variability than that observed in real cells.

systematic differences in $C_{V}$ between cells in MT and V1; we did not pursue this question further.

Both sets of data show an increase of $C_{V}$ values from the shortest mean ISIs measured $(3 \mathrm{msec})$ up to longer ISIs $(10-15$ $\mathrm{msec})$. As discussed in the preceding section, the possible drop in $C_{V}$ at high values of the ISI ( $30 \mathrm{msec}$ ) is most likely a measurement artifact that underestimates $C_{V}$ when both $C_{V}$ and $\Delta t$ are large (Fig. 4). The drop for low values of the ISI (high firing frequencies), on the other hand, is a real effect and is in agreement with standard models (see below). While most histograms did not have sufficient counts to justify a functional fit, $C_{V}$ values near unity are characteristic of the exponential ISI distributions of a Poisson process, the most random type of spike distribution possible.

\section{Variability in the number of spikes}

As a further test of the variability of these spike trains, we analyzed the number of spikes $S_{j}$ occurring in a train in response to a specific and constant stimulus. We plotted the variance in the number of action potentials per stimulus presentation $\left(\sigma_{S}^{2}\right)$ against the average number of spikes $S_{\text {avg }}$ for the same nonbursting trains studied above. As is evident from the log-log plots in Figure $5, \sigma_{S}^{2}$ is scattered widely about the mean spike number in area $\mathrm{V1}$, and equal to or above the mean spike number in area MT. In the case of our large number of MT neurons, we found that the response variance in MT scales approximately as $\sigma_{S}^{2} \propto S^{5 / 4}$. In the case of a pure Poisson process, the variance in the number of events is equal to the mean. Thus, their ratio should be unity, independent of firing rate.

\section{Analytical Models}

In this and the following sections, we will attempt to account for this high degree of variability using simple analytical models of the spiking process.

\section{Integrate-and-fire neuron}

A neuron is most simply modeled as a single capacitance with an associated membrane potential $V$, which can be stepwise 
increased by pulses of constant charge, each pulse incrementing $V$ by a fixed amount. When $V$ exceeds a certain threshold voltage, the model neuron produces an output spike and immediately resets its voltage to the resting value $V=0$ (for references to this "integrate-and-fire" model, see Tuckwell, 1989; see also Knight, 1972). The neuron's "threshold" can be expressed in terms of the number of pulses (an integer $N_{\mathrm{th}} \geq 1$ ) necessary to bring the cell from rest to discharge.

We further assume that these spike-like pulses arrive completely randomly in time (Poisson distributed), with a mean rate of arrival $R$. Throughout our study, we assume that the synaptic input pulses are drawn from a Poisson distribution (we will reexamine this crucial assumption at the end of the discussion). This randomness assumption is consistent with superposing many independent but possibly non-Poisson input spike trains (Cinlar, 1972). This randomness is also consistent with the stochastic nature of synaptic transmission: if each synaptic site has a probability $p(p<1)$ of transforming a Poisson action potential into an EPSC, the resulting sequence of EPSC's will still be Poisson (but with a lower mean rate $p R$ ). Large numbers of spike trains can be superposed to produce more variability than a Poisson train only if the individual spikes from the various trains are temporally synchronized (a highly nonrandom but important situation, which we consider in the Network Effects section).

This kind of integrating neuron gives one output pulse for every $N_{\text {th }}$ input pulse. As a result, the ISI of the output is just the sum of the $N_{\mathrm{th}}$ interpulse intervals between the cell's previous spike and the final pulse that triggered the cell's response. With Poisson-distributed pulses, the probability distribution $p(\Delta t)$ of their sum-and hence the predicted shape of the output ISI histogram - is a gamma function of order $N_{\mathrm{th}}-1$ (Tuckwell, 1989),

$$
p(\Delta t) \propto(R \Delta t)^{N_{\mathrm{th}}-1} \exp (-R \Delta t) .
$$

Integration of this function over $\Delta t$ yields the mean and standard deviation, namely,

$$
\overline{\Delta t}=\frac{\int_{0}^{+\infty} \Delta t p(\Delta t) d \Delta t}{\int_{0}^{+m} p(\Delta t) d \Delta t}=\frac{N_{\mathrm{th}}}{R}
$$

and

$$
\sigma_{د t}^{2}=\frac{\int_{0}^{+\infty}(\Delta t-\overline{\Delta t})^{2} p(\Delta t) d \Delta t}{\int_{0}^{+\infty} p(\Delta t) d \Delta t}=\frac{N_{\mathrm{th}}}{R^{2}},
$$

which give

$$
C_{i}=\frac{\sigma_{د t}}{\overline{\Delta t}}=\frac{1}{\sqrt{N_{\mathrm{th}}}}
$$

Thus, for this "integrate-and-fire" model of a nerve cell with independent synaptic input, $C_{V}$ is independent of firing rate, since both $\Delta t$ and $\sigma_{\Delta}$ scale inversely with $R$. To apply this model to real cells, we suppose that an approximate threshold depolarization for a pyramidal cell is $20 \mathrm{mV}$ from rest to firing, and typical depolarizations for a single excitatory EPSP onto a pyramidal cell (in rat visual cortex) are in the range of $0.05-0.5$ $\mathrm{mV}$ per excitatory input (reported for detectable monosynaptic contacts among pyramidal cells in rat cortex by Mason et al., 1991). These admittedly crude values yield $N_{\mathrm{th}} \geq 40$ EPSPs, and $C_{l} \leq 0.16$; that is, the cell should spike rather regularly. The fact that Equation 12 predicts $C_{V}<0.5$ for all threshold values $N_{\text {th }}>3$ pulses (while empirically $C_{V}>0.5$ ) constitutes the central difficulty this article sets out to explore.

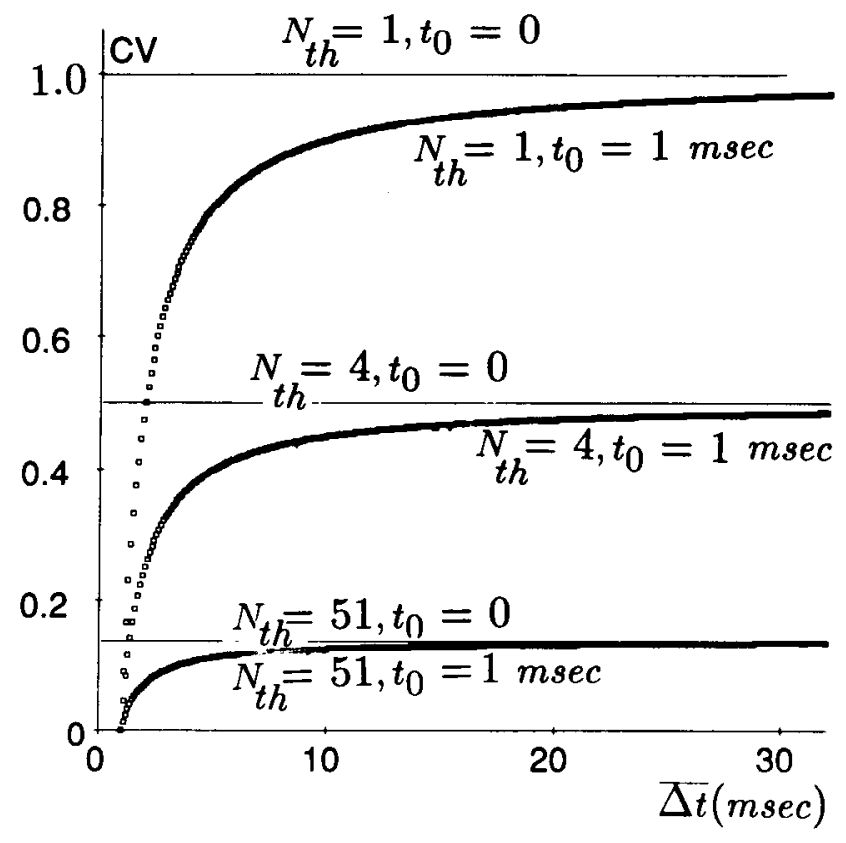

Figure 6. Comparison of $C_{V}$. from integrator models. Straight lines represent predictions of $C_{k}$ for a neuronal integrator that fires after receiving $N_{\mathrm{th}}$ randomly timed input impulses. The curves show $C_{1}$ for such a model, modified to account for an absolute refractory period $t_{0}$ $=1.0 \mathrm{msec}$ (curves computed using a different refractory period would have a similar shape, always crossing the $\overline{\Delta t}$ axis at $t_{0}$ ). Note that $C_{\mathrm{r}} \leq$ $1 / \sqrt{N_{\mathrm{th}}}$ for all models, such that $C_{\mathrm{l}}$ is quite small (output spikes are regular) for large values of $N_{\mathrm{th}}$.

\section{Refractory period}

Real nerve cells, however, cannot fire a second action potential immediately after a first, since the sodium channels must deactivate and be repolarized before further activation. As a result, the cell undergoes a short "absolute refractory period," during which it cannot be discharged, followed by a much longer "relative refractory period," during which it is difficult to discharge.

A convenient oversimplification to this case is to modify the perfect integrate-and-fire model by the addition of an absolute refractory period $t_{0}$ ("dead time") immediately after resetting, during which the neuron is entirely inactive and after which it resumes normal function. Because the same time $t_{0}$ is added to each and every ISI $\Delta t$, the net effect is to shift the entire ISI histogram (Eq. 9) rightward by $t_{0}$ :

$$
\begin{aligned}
& p(\Delta t) \propto\left[R\left(\Delta t-t_{0}\right)\right]^{N_{\mathrm{th}}-1} \exp \left[-R\left(\Delta t-t_{0}\right)\right], \quad \text { for } \Delta t>t_{0}, \\
& p(\Delta t)=0, \quad \text { for } \Delta t \leq t_{0} .
\end{aligned}
$$

This refractory period now gives the neuron a characteristic time scale, so we cannot expect it to have identical statistics at all firing rates. In particular, the value for $\sigma_{\Delta t}(\mathrm{Eq} .11)$ now depends on $\overline{\Delta t}-t_{0}$ rather than on $\overline{\Delta t}$, so that the new value of $C_{l}$ does depend on the mean ISI:

$$
C_{r}=-\frac{1}{\sqrt{N_{\mathrm{th}}}}\left(\frac{\overline{\Delta t}-t_{0}}{\overline{\Delta t}}\right) \leq \frac{1}{\sqrt{N_{\mathrm{th}}}} .
$$

The refractory period has little effect for $\overline{\Delta t} \gg t_{0}$ (since in this case $C_{\mathrm{V}} \approx 1 / \sqrt{N_{\mathrm{th}}}$ ), but as $\overline{\Delta t} \rightarrow t_{0}$ the output spike train becomes extremely regular $\left(C_{1} \rightarrow 0\right)$, regardless of $N_{\text {th }}$ (Fig. 6). In general, the $C_{r}$ for this simple model of a refractory period is always less than the $C_{V}$. of the standard integrate-and-fire model (Eq. 


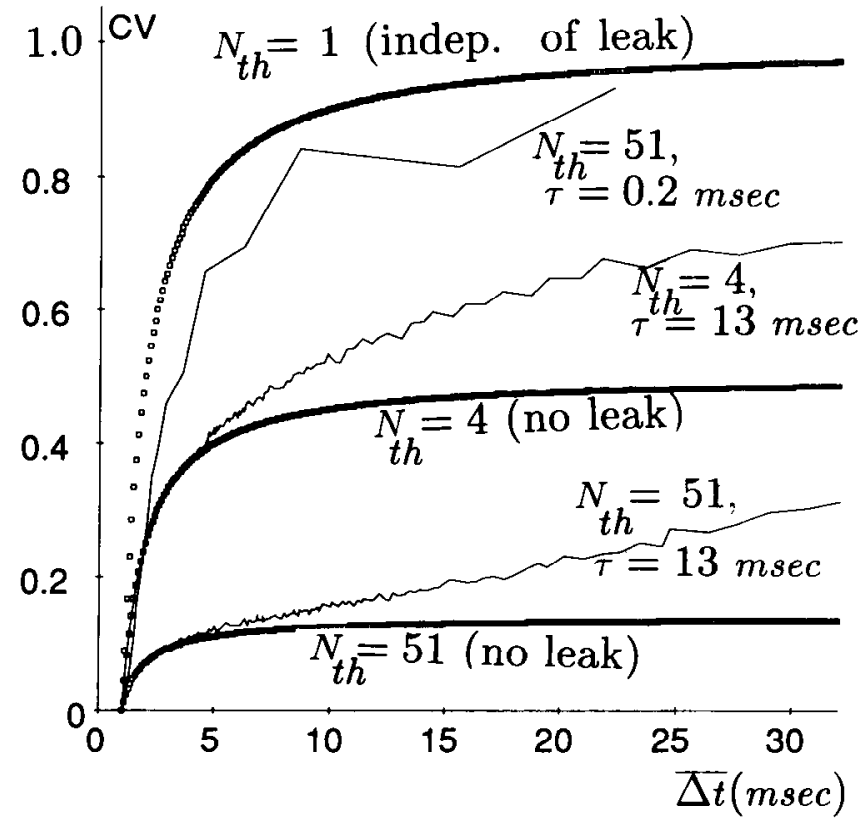

Figure 7. Comparison of leaky and nonleaky integrator models. Squares show $C_{1}$ for the nonleaky integrator model with absolute refractory period $t_{0}=1.0 \mathrm{msec}$. Crooked lines show simulations of the leaky integrator for three different values of membrane time constant. The leak term has no effect on $C_{r}$ for the $N_{\mathrm{th}}=1$ integrator, but raises $C_{V}$. if $N_{\mathrm{th}}>1$. Only for small values of $\tau(\ll \overline{\Delta t})$ does $C_{l}$. approach unity.

12). This result is easy enough to understand: the very fastest the cell can fire is once every $t_{0}$, when the integration period is much shorter than $t_{0}$ and contributes little variation. The sudden drop in $C_{1}$. in the fastest-firing monkey cells for very small values of $\Delta t$ suggests that this effect-rather than $N_{\mathrm{th}}-$ is the dominant influence in the regularity of those cells (see Fig. 9; we chose a conservative $t_{0}=1.0 \mathrm{msec}$ for all comparisons, because a larger $t_{0}$, leading to an even lower value of $C_{b}$, would only increase the gap between the predicted $C_{l}$. and monkey data).

\section{Leaky integate-and-fire neuron}

It is well known that depolarizations do not persist forever, but that perturbations of membrane voltage tend to decay toward the resting potential (we assume $V_{\text {resi }}=0$ for mathematical simplicity). The simplest physical model of this current "leak" is the inclusion of a passive membrane conductance $\left(1 / R_{m}\right)$, in parallel with the capacitance of the perfect integrator. [This "leaky" or "forgetful" integrator is described in detail by Stein (1967a) and Knight (1972).] The "leaky integrator" has a decay time constant $\tau=R_{m} C_{m}$, giving a behavior between discharges of

$$
\frac{d V}{d t}=-\frac{V}{\tau}+\text { pulses }
$$

The passive decay inherent in Equation 15 is a simplification of the action of active, voltage-dependent conductances in the membrane of the soma and proximal dendrites. However, it does allow us to capture the essential qualitative aspects of temporal decay. But despite decades of effort (Tuckwell, 1989), the ISI histogram and $C_{V}$ for even this simple model are not available in closed form. Our predictions for the $C_{t}$ of this model come from numerical simulation of Equation 15, using a realistic value for the membrane time constant of $\tau=13 \mathrm{msec}$ in the presence of random input pulses (Mason et al., 1991).

Qualitatively, the leak term has little effect on the $C_{r}$ at high firing rates $(\overline{\Delta t} \leq \tau)$, because there is not sufficient time to discharge the capacitance significantly through the leak before the threshold $N_{\mathrm{th}}$ is reached. But at very low firing rates ( $\overline{\Delta t} \gg$ $\tau)$ the output spikes are nearly random $\left(C_{l} \approx 1\right)$ because the neuron operates as a "coincidence detector" for occasional bursts of EPSPs. In this mode, the membrane potential $V$ "forgets" when the last firing occurred, so that the subsequent firing time is virtually independent of the previous time; that is, the model neuron's output nearly approximates a Poisson process. Thus, the neuron smoothly interpolates between a low $C_{r}$ (given by Eq. 14) and the maximum possible $C_{1}=1$ as the output ISI increases.

A plot of $C_{r}$ against $\overline{\Delta t}$ for this model for various levels of thresholds $N_{\mathrm{th}}$ illustrates the conflict between the predicted and our observed results (Fig. 7). These results show that $C_{1}>0.5$ only occurs for $\overline{\Delta t}>10 \tau$ or $N_{\mathrm{th}} \leq 3$ (low threshold). The case of a small $\tau$, such that $\overline{\Delta t} \gg \tau$, corresponds to the situation wherc a large membranc lcak cxists in the ccll's membranc. The conflict between theory and data is greatest for the fastest-firing cells ( $\overline{\Delta t}<\tau \approx 13 \mathrm{msec}$ ); in that regime the leaky-integrator prediction is approximately given by Equation 14. A contour plot for $C_{V}$ as a function of $N_{\mathrm{th}}$ and $\tau$, using a fixed output spike rate $R=1 / \Delta t=200 \mathrm{~Hz}$ and absolute refractory period $t_{0}=1$ msec, is shown in Figure 8. It is evident that in order to achieve high variability (i.e., $C_{1}>0.7$ ) at these high rates (which are comparable to those in our faster cells), $\tau$ has to be a fraction of a millisecond, or $N_{\mathrm{th}}$ must be only 1 or 2! In fact, the model best fitting the monkey data is that for a neuron that performs no temporal integration, having $N_{\mathrm{th}}=1$ (Fig. 9).

\section{Realistic parameters and modifications}

In light of the serious discrepancy between the monkey data and the simple theory for random input to an integrator, we investigated several modifications to the theory. The modifications, like the foregoing analysis, are only approximate. When possible they are given as correction coefficients to the perfect integrator with refractory period (Eq. 14). The resulting patchwork of approximations outlines the major probable influences of these various biophysical modifications on ISI variability. We reserve the detailed equations for the appendix and outline here the qualitative effects.

\section{Variable EPSP magnitude}

Our previous model includes a crude approximation of random excitatory postsynaptic potentials (EPSPs) of constant amplitude and arriving randomly in time. However, the magnitude of EPSPs is expected to vary greatly, depending on their location on the dendritic tree, quantal fluctuations, and so on. Clearly, including random EPSP amplitude as an additional source of variability will increase the variability in the cell's synaptic input and hence its firing. Recent in vitro two-electrode intracellular recordings in pyramidal cells in rat visual cortex have shown that the variation in amplitude of unitary EPSPs (from different synapses) is nearly equal to the average amplitude of these unitary EPSPs (0.05-0.5 mV; Mason et al., 1991). Even after incorporating such variable-sized synaptic input into the perfect 


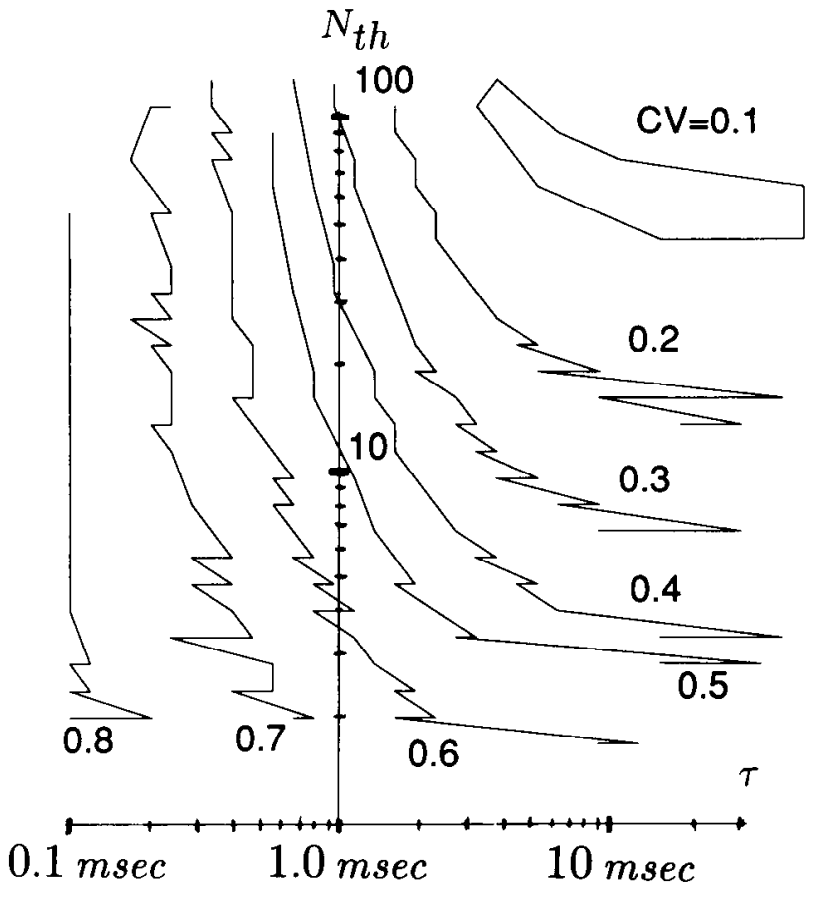

Figure 8. Contour plot of $C_{1}$ for leaky integrator. Simulations of the leaky integrator model for discrete values of $\tau$ and $N_{\mathrm{th}}$ (with refractory period $t_{0}=1.0 \mathrm{msec}$ ) give the $C_{V}$, values shown when the mean output ISI is $\overline{\Delta t}=5 \mathrm{msec}$ (corresponding to a mean firing rate of $200 \mathrm{~Hz}$ ). The jagged contours result from simulating $N_{\mathrm{th}}$ and $\tau$ at discrete values. Accepted biological parameters (e.g., $N_{\mathrm{th}}>10, \tau>5 \mathrm{msec}$ ) predict low $C_{1}$ values (upper right region); the $C_{1}$. values observed in monkey would require either $N_{\mathrm{th}}<3$ or $\tau<1 \mathrm{msec}$ (lower left region).

integrate-and-fire model, $C_{1}$ only increases from its old value of $\sqrt{1 / N_{\mathrm{th}}}$ to

$$
C_{V}=\sqrt{2 / N_{\mathrm{th}}}
$$

(derived in Stein, 1967a). This factor of $\sqrt{2}$ is not sufficient to remove the discrepancy between the model and our monkey data.

\section{Finite EPSC width}

The model above assumes that EPSCs are instantaneous pulses, which can carry variability at arbitrarily high temporal frequencies. But even the fastest unitary synaptic currents last between 1 and $2 \mathrm{msec}$ (in the case of fast, non-NMDA, glutamergic synapses; Hestrin et al., 1990), blurring the total synaptic current and reducing its variability. Due to this smoothing effect, we conclude in the Appendix that $C_{V}$ in a typical non-NMDA case would be reduced by a factor of 2 below the value predicted above, thus compounding the discrepancy between predicted and observed $C_{1}$, values.

\section{Adaptation and hyperpolarizing currents}

The simplified model above does not take hyperpolarizing currents (such as the $I_{\mathrm{AHP}}$ "spike adaptation" potassium current, or slow $\mathrm{GABA}_{\mathrm{B}}$ synaptic inputs) into account. The main predicted effect of such currents is to reduce the firing rate, by effectively canceling a portion of the depolarizing current. As the rate reduces, the mean ISI increases and allows time for more EPSPs to impinge on the cell before each firing. Thcsc

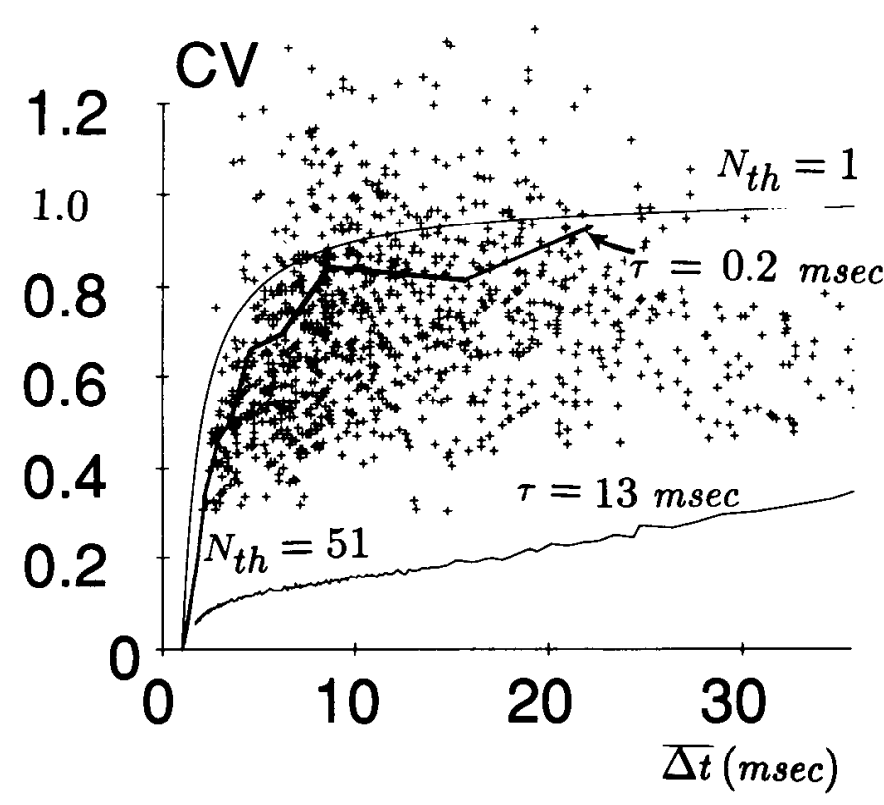

Figure 9. Comparison of macaquc cortical ncuron variability and lcakyintegrator model. Scattered crosses show $C_{i}$ for macaque cortical neurons $\left(C_{V}\right.$ was pooled from Fig. 3). The lower curve shows the simulated leaky-integrator model with parameter values in the accepted range $\left(N_{\mathrm{th}}\right.$ $=5 \mathrm{1}, \tau=13 \mathrm{msec}$, and $t_{0}=1.0 \mathrm{msec}$ ). The middle curve shows the same simulation, still with $t_{0}=1.0$ but $\tau=0.2 \mathrm{msec}$, a much shorter decay time than usually accepted for pyramical cells. The upper curve shows the theoretical upper bound on $C_{1}$ for a pure Poisson spike train with "dead-time" $t_{0}=1.0 \mathrm{msec}$. The observed $C_{1}$ of macaque cortical cells lies much closer to the maximum possible than it does to the $C_{1}$. predicted by a neuron model that performs significant temporal integration.

extra EPSPs carry with them some added variability, so that $C_{1}$. will increase above the predicted value as the ISI increases. This effect (which is observed in the monkey cells and the compartmental simulation following) is not strong enough to account for the discrepency between the model and the monkey data; in addition, it cannot change the predicted $C_{V}$ for spikes during the early, nonadapted portion of the cell's response.

\section{Compartment Models}

Even with the modifications discussed above, we had to make a certain number of risky simplifications. For instance, we did not account for the complex dynamics of cellular cxcitability or its known dendritic morphology, nor did we include a treatment of the effect of fast synaptic inhibition. To answer these criticisms, we studied the firing properties of a biophysically very detailed model of a single cortical pyramidal cell using conventional compartmental techniques.

\section{Biophysical modeling of a cortical pyramidal cell}

We simulated the firing properties of a layer $\mathrm{V}$ pyramidal cell (see drawing in Fig. 10) from primary visual cortex, whose detailed morphology was reconstructed following intracellular filling with HRP during in vivo experiments in the anesthetized, adult cat (Douglas et al., 1991). Its dendritic tree was described as a list of 186 one-dimensional cables of specified length and diameter, all of which were assumed to be passive. The cell body contained seven voltage- and calcium-dependent currents; a fast, classical sodium current, $I_{\mathrm{Na}}$ (with peak conductance per mem- 


$\begin{aligned} & \text { Table 1. Predicatability of number of spikes in simulated pyramidal } \\
& \text { cell }\end{aligned}$
\begin{tabular}{lccc} 
Avg. EPSP & $\begin{array}{l}\text { Spikes } S \text { in } \\
\text { a train }\end{array}$ & Trains \\
Simulation & $47 \mathrm{kHz}$ & $104.6 \pm 1.5$ & 100 \\
\hline "Barely plausible" & $26 \mathrm{kHz}$ & $66.5 \pm 1.5$ & 100 \\
& $16 \mathrm{kHz}$ & $43.7 \pm 1.5$ & 100 \\
& $8.5 \mathrm{kHz}$ & $23.7 \pm 1.3$ & 248 \\
& $400 \mathrm{kHz}$ & $42.6 \pm 0.5$ & 50 \\
& $232 \mathrm{kHz}$ & $32.1 \pm 0.4$ & 50 \\
& $103 \mathrm{kHz}$ & $21.0 \pm 0.5$ & 50
\end{tabular}

The compartmental-model simulation using passive dendrites produced a very predictable number of spikes for a given average EPSP rate. Shown are EPSP rates, the spike number $S$ (averaged over all trains at that rate), and the number of trains simulated at that rate. Note that the variability in spike number is far smaller than the $1 / \sqrt{S}$ variation expected for Poisson-distributed spikes.

brane area of $\left.200 \mathrm{mS} \cdot \mathrm{cm}^{-2}\right)$; a slow, noninactivating sodium current $I_{\mathrm{Na.s}}\left(1 \mathrm{mS} \cdot \mathrm{cm}^{-2}\right)$; an L-type calcium current, $I_{\mathrm{Ca}}(0.2$ $\left.\mathrm{mS} \cdot \mathrm{cm}^{-2}\right)$; and four potassium currents [delayed rectifier $I_{\mathrm{DR}}$ $\left(120 \mathrm{mS} \cdot \mathrm{cm}^{-2}\right)$, transient $I_{\mathrm{A}}\left(1 \mathrm{mS} \cdot \mathrm{cm}^{-2}\right)$, calcium-dependent $I_{\mathrm{K}(\mathrm{Ca})}\left(45 \mathrm{mS} \cdot \mathrm{cm}^{-2}\right)$; and a noninactivating $I_{\mathrm{M}}$ current $(0.6 \mathrm{mS}$. $\left.\mathrm{cm}^{-2}\right)$ ]. These currents were modeled using Hodgkin-Huxleylike rate constants (Bush and Douglas, 1991). This model was studied in detail by Ö. Bernander (Bernander et al., 1991), using the very efficient single neuron simulator NEURON, provided by Hines (1989). The somatic spiking threshold as well as the $f-I$ curve for the simulated cell matched those recorded intracellularly in vivo (for more details, see Bernander et al., 1991).

We used an effective passive specific membrane resistance of $26,000 \Omega \cdot \mathrm{cm}^{2}$ throughout the cell. Under these conditions, the somatic membrane potential stabilized at $-75 \mathrm{mV}$, with a spiking threshold of about $-48 \mathrm{mV}$, a somatic passive time constant of $30 \mathrm{msec}$, and an input resistance of $42 \mathrm{M} \Omega$, corresponding to a good and stable intracellular recording from in vivo cat pyramidal cells (Douglas and Martin, 1991). All synaptic inputs were modeled as transient increases in the membrane conductance, $g_{\text {syn }}(t) \propto t \exp \left(-t / t_{\text {peak }}\right)$ with $g\left(t_{\text {peak }}\right)=g_{\text {peak }}$, in series with the synaptic reversal battery $E_{\text {syn }}$.

\section{"Conventional" and "barely plausible" simulations}

We then ran two distinct sets of simulations to study the temporal variability of the discharge of this pyramidal cell. In one case ("conventional" or "c." simulation), we used synaptic conductance amplitudes and distributions in rough agreement with experimental findings, while for a second set of simulations ("barcly plausible" or "b.p.") we pushed these parameters to the limits of the accepted ranges in order to increase the temporal variability. The b.p. simulation therefore reflects the outer range of temporal variability compatible with a passive dendritic membrane and independent synaptic inputs.

Both sets of simulations included both excitatory as well as inhibitory synaptic input. In the "conventional" model, simulated input from a basket cell activated 30 synchronous somatic $\mathrm{GABA}_{\mathrm{A}}$ synaptic events $\left(g_{\text {peak }}=0.1 \mathrm{nS} ; t_{\text {peak }}=5 \mathrm{msec} ; E_{\text {rev }}=\right.$ $-70 \mathrm{mV}$ ). All 30 synapses were randomly but jointly activated at the average rate of $450 \mathrm{~Hz}$. The maximum, saturated $\mathrm{GABA}_{\mathrm{A}}$ conductance at one synapse (due to several consecutive events) was set to $0.5 \mathrm{nS}$. These 30 basket cell synapses contributed a mean somatic conductance increase of $10 \mathrm{nS}$ [this value is in the range reported by Douglas and Martin (1991), for the total amount of inhibition].

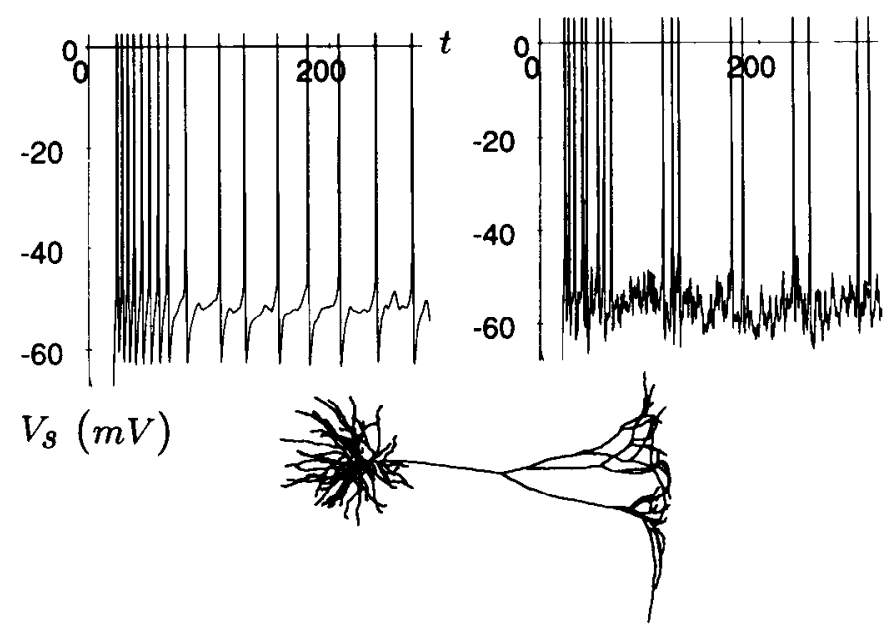

Figure 10. Somatic potential of a simulatcd pyramidal cell. A compartmental model (with passive dendrites) of a reconstructed striate cortex layer $\mathrm{V}$ pyramidal cell (drawing) produced the somatic voltage traces shown upon exposure to random EPSPs and IPSPs. Left, Somatic voltage in the c. simulation, with $t_{\text {peak }}=1.5 \mathrm{msec}, g_{\max }=0.5 \mathrm{nS}$, with excitatory synapses distributed randomly throughout the dendritic tree. Right, Simulated somatic voltage in the b.p. simulation, which used parameters at the edge of accepted ranges to create the most variability possible: a fast and strong $\operatorname{EPSP}\left(t_{\text {peak }}=0.3 \mathrm{msec}, g_{\text {peak }}=10 \mathrm{nS}\right)$ and all synapses located on the apical dendrite $60 \mu \mathrm{m}$ from the soma. Note the adaptation in firing frequency following onset of the "stimulus."

For the b.p. model, the number of these inhibitory synapses was kept constant, but $g_{\text {peak }}$ was tripled to $0.3 \mathrm{nS}$ and the synaptic conductance saturation was eliminated (allowing consecutive synaptic inputs to add in time), so that the net inhibitory conductance change at the soma fluctuated around $60 \mathrm{nS}$. The resting potential at the cell body stabilized (as before) at around $-72 \mathrm{mV}$. By temporarily removing all the active currents at the cell body, we estimated the resulting average input resistance and passive time constant during the inhibitory synaptic barrage as $R_{N}=11 \mathrm{M} \Omega$ and $\tau=7 \mathrm{msec}$ for the b.p. cell, and $R_{N}=30$ $\mathrm{M} \Omega$ and $\tau=13 \mathrm{msec}$ for the c. model. Since the intracellular recorded values of $R_{N}$ and $\tau$ for the reconstructed cell were 23 $\mathrm{M} \Omega$ and $20 \mathrm{msec}$, respectively (Bernander et al., 1991), the c. case study represents a realistic cortical cell simulation. As mentioned above, the b.p. model will push $C_{l}$ toward higher values by decreasing the effective membrane time constant.

Excitatory input was provided to the c. model by placing excitatory synapses at 20 difference locations throughout the basal and apical tree. The excitatory synapses were assumed to be voltage independent of the AMPA or non-NMDA type ( $E_{\text {syn }}$ $=0 \mathrm{mV}$; mean conductance $\bar{g}_{\text {peak }}=0.5 \mathrm{nS} ; t_{\text {peak }}=1.5 \mathrm{msec}$ ). Furthermore, since individual synaptic amplitudes may vary, the value of $g_{\text {peak }}$ for each synaptic event was chosen from an exponential probability $P(g) \infty \exp (-g / \bar{g})$, so synaptic events had a high variability in amplitude, even events occurring at the same location. These values led to somatic EPSPs ranging from a mean of $0.4 \mathrm{mV}$ peak potential and $4 \mathrm{msec}$ rise time for the most proximal synapse to about $0.5 \mu \mathrm{V}$ for the most distal one. These values are within the range reported in rat visual cortex slice pyramidal cells for unitary EPSPs evoked by stimulating a single presynaptic pyramidal cell (Mason et al., 1991), and with values obtained with spike-triggered averaging of EPSPs in cat visual cortex (Komatsu et al., 1988).

In order to reduce dendritic attenuation and temporal smoothing in the b.p. model, and thereby increase $C_{1}$, all ex- 


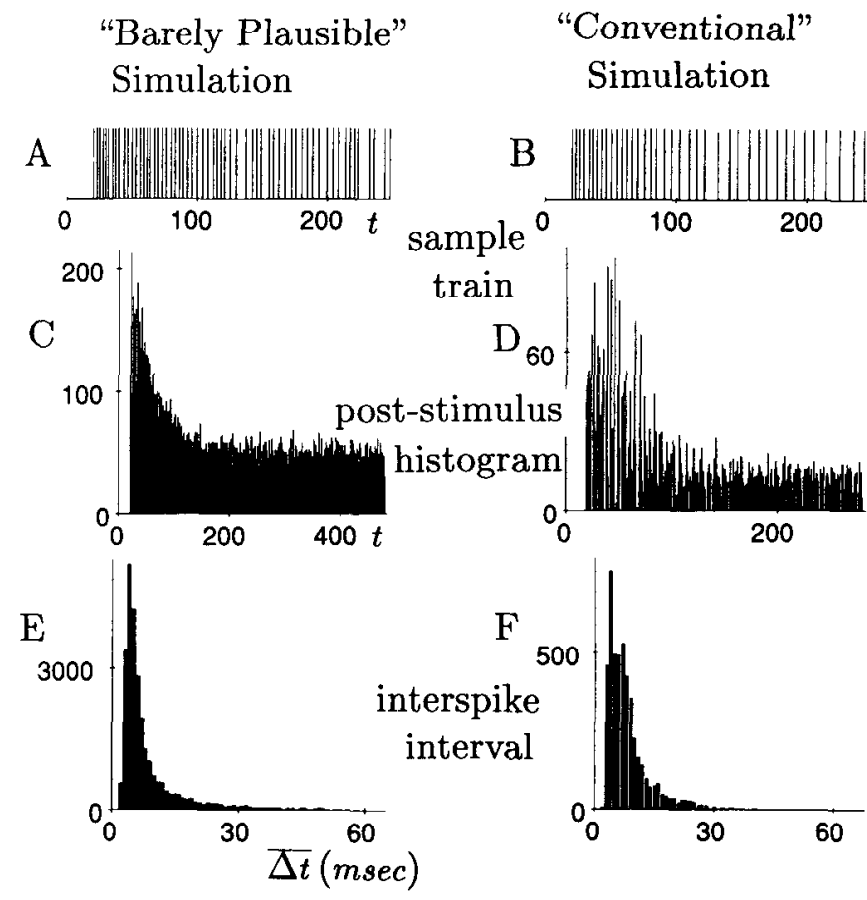

Figure 11. Firing statistics of detailed pyramidal cell simulation. Our compartmental model produced the spike trains shown upon exposure to random EPSPs. The left column used b.p. parameters to simulate a more variable output; the more regular simulation at right used c. parameters. $A$ and $B$. Sample spike trains from each simulation. $C$ and $D$, PSTHs from the same simulations. The prominent millisecond structure in the PSTH (especially the first $50 \mathrm{msec}$ ) results from the highly regular simulated trains, in which early spike times are well correlated with the onset of stimulation. Although the simulation parameters were fairly conventional, the highly regular spiking they produced was not observed in most cortical neurons. $E$ and $F$, ISI histograms from the same neuron. The broadness of these histograms arises from the combination of different mean firing rates in one histogram, an effect eliminated in the multihistogram analysis of $C_{2}$.

citatory synapses were placed on the proximal apical dendrite only $60 \mu \mathrm{m}$ away from the soma. At each synapse $t_{\text {peak }}=0.3$ msec and $\bar{g}_{\text {peak }}=10 \mathrm{nS}$, giving rise to a very large somatic EPSP (mean depolarization, $1.6 \mathrm{mV}$ ) within $1 \mathrm{msec}$. This simulation only required the simultaneous occurrence of 19 of these "giant" EPSPs on average to bring the cell from rest to the firing threshold.

Synapse activation times were random (with a fixed probability per unit time), with average rates chosen to yield output spike rates comparable to those analyzed from the monkey (40$200 \mathrm{~Hz}$ ). This required total excitatory synaptic activation rates of $100-400 \mathrm{kHz}$ for the c. model and $8.5-47 \mathrm{kHz}$ for the b.p. simulation. The integration step size used by NEURON was $d t=$ $0.1 \mathrm{msec}$, with random synaptic activation summed over 0.02 msec subintervals. The simulations generated nearly 700 spike trains of 230 (c.) or $470 \mathrm{msec}$ (b.p.) duration. In order to avoid any systematic biases, we analyzed these spike trains with the exact same normalization method outlined in the Parameters and Normalization Procedure section. Table 1 gives the values of the EPSP rates used as well as the number of spikes produced, and Figure 10 gives one example of a $200 \mathrm{msec}$ excerpt of the somatic potential for typical c. and b.p. simulations. Figures 11-14 show the resulting spike trains, histograms, and $C_{l^{\prime}}$.

In order to test whether these low $C_{V}$, results depended on the details of our voltage-dependent somatic currents, we introduced two modifications to the detailed kinetic schemes of the
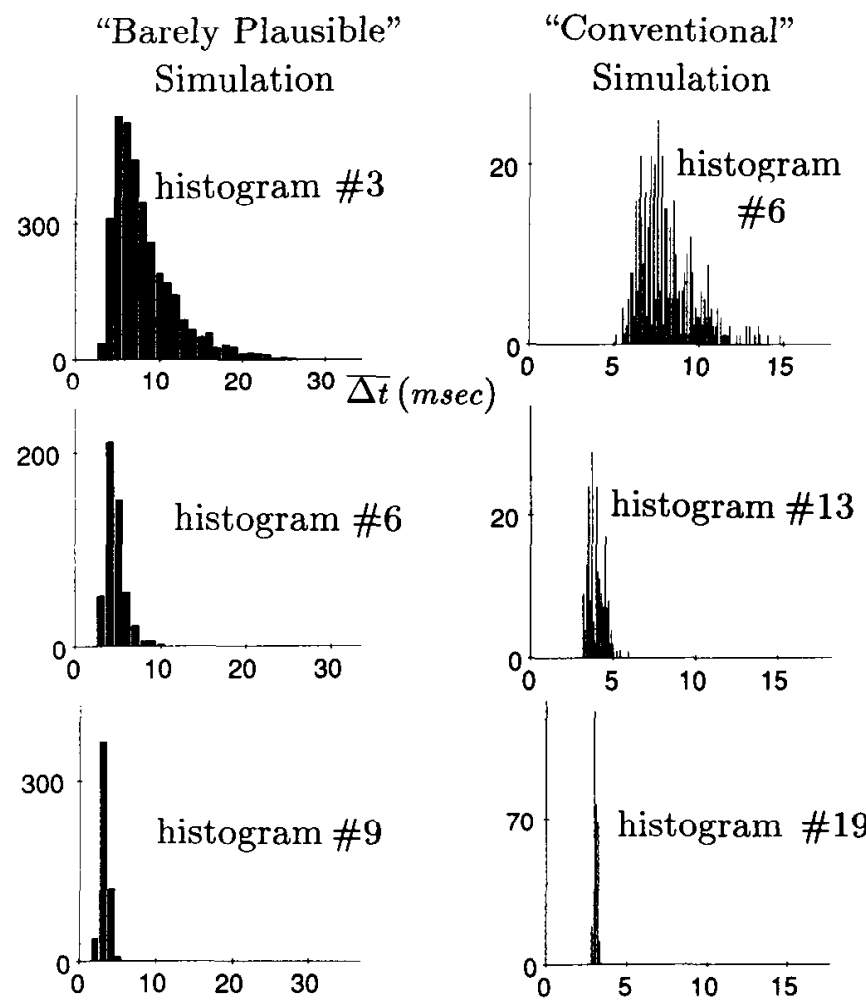

Figure 12. ISI histograms of simulated spike trains. At the left are histograms using $1.0 \mathrm{mscc}$ bins, with data from the b.p. compartmental model, analyzed as described in section 2 . At the right are histograms from the c. model (here shown as analyzed using $0.1 \mathrm{msec}$ bins and 20 histograms for greater resolution). Note that both models have much narrower histograms than the macaque data, especially at short ISI values (high fring rates), reflecting the unnaturally high regularity of these simulated trains.

fast sodium current responsible for the action potential in the b.p. simulation. One modification lowered the firing threshold by lowering the midpoint voltage $V_{1 / 2}$ at which the steady-state value of the sodium activation particle $\left(m_{\infty}\right)$ was half its maximum (i.e., 0.5). When $V_{1 / 2}$ was lowered from $-40 \mathrm{mV}$ to -50 $\mathrm{mV}$, the firing threshold was reduced proportionately, but the ccll's firing variability in response to random synaptic input only increased slightly (as would be expected in the integrator model, for which a lowered value of the spike threshold gives rise to a lower value of $N_{\mathrm{th}}$ and hence a slightly higher $C_{\mathrm{v}}$ ).

In an alternative modification, the initial Hodgkin-Huxleylike currents $\left(I_{\mathrm{Na}}, I_{\mathrm{DR}}\right)$ were kept, while all other active currents were blocked, so that the simulated cell's $f-I$ curve showed the steep onset of spiking typical of a Hodgkin-Huxley-like system. The response of this model to the same random synaptic events tested above only differed in an absence of adaptation; the variability remained the same (not shown).

\section{Simulation results}

The very regular spike trains from these simulations led to low $C_{V}$, values, in particular at high firing rates: for $\overline{\Delta t}<5 \mathrm{msec}$ (i.e., firing rates above $200 \mathrm{~Hz}$ ) $C_{V}<0.2$, rising to 0.65 (b.p.) or 0.3 (c.) for $\overline{\Delta t}>10 \mathrm{msec}$ (Fig. 13). Thus, they fail to reproduce by a large margin our experimentally measured variability at high firing rates.

One indicator of the regularity of the generated action potential traces is that different simulated spike trains sharing a com- 


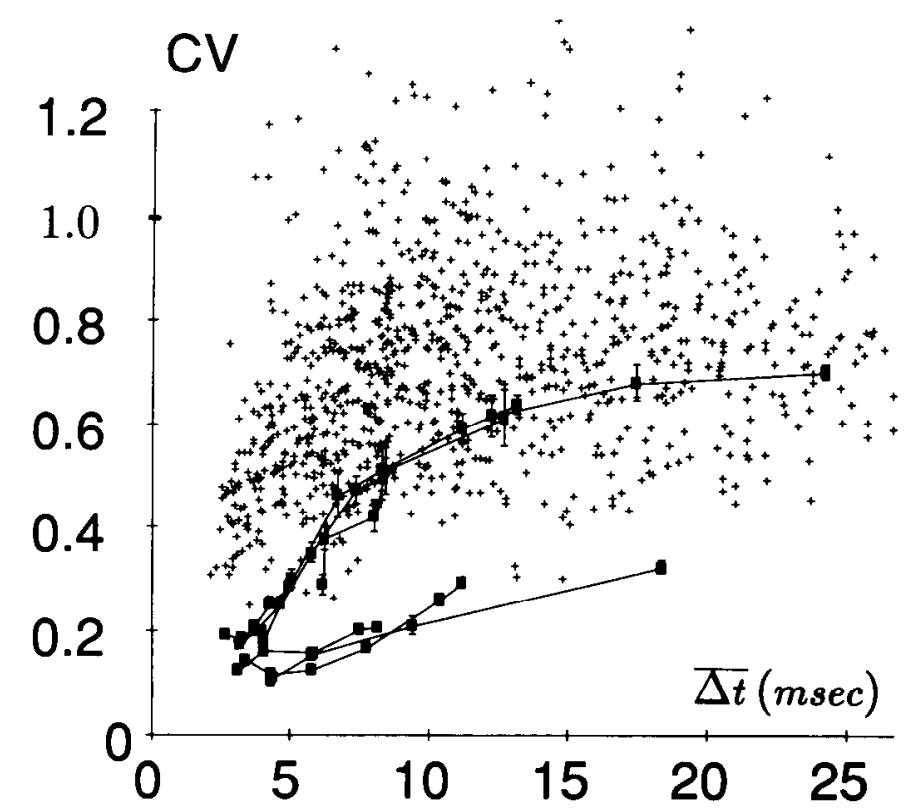

Figure 13. Comparison of $C_{\mathrm{r}}$. values from compartmental simulations with macaque data. Scattered pluses are $C_{V}$, from areas V1 and MT. All sets of connected points represent simulations with random EPSP input to our detailed model of a reconstructed pyramidal cell. All data shown were analyzed with the same method (using $1.0 \mathrm{msec}$ bins). The upper sets of squares are from four different EPSP rates in the b.p. simulations, with fast EPSP duration, high $g_{\text {peak }}$, and all synapses near the soma. The lower sets of squares resulted from three EPSP rates in the c. model, with slower and smaller synapses distributed over the dendritic tree. The $C_{1}$ exhibitcd by this model is much lower than for the b.p. case, because more EPSPs (130) were needed to fire the cell, and because high-frequency variation in the input is attenuated by the dendritic tree and the slow $t_{\text {pcak }}$ of the synapses. Note that for ISIs less than $10 \mathrm{msec}$, both simulations give $C_{1}$ values far less than those observed in monkey.

mon average input EPSP rate (but with distinct time structures due to the random synaptic activation times) had virtually identical total numbers $S_{j}$ of spikes. For a fixed excitatory input rate,

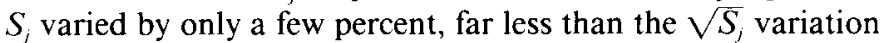
expected of a totally random point process or observed for our monkey data (see Table 1, Fig. 5).

Another indicator of the extreme spiking regularity of the $\mathrm{c}$. simulation was the presence of prominent peaks on the PSTH long after stimulus onset (Fig. 11D); the trains were so regular that a single spike's occurrence could be predicted to a few milliseconds even $150 \mathrm{msec}$ after the first spike fired! For these simulations of a passive-dendrite pyramidal cell, there exists a difference of over 1 order of magnitude between the expected and the measured variability.

\section{Comparison of compartmental and analytical model}

It has been argued that the leaky integrator is such a simplified model of a real neuron-especially at high firing rates - that little can be learned from it. But our simulations do not support this view.

While researchers usually believe that cortical neurons integrate synaptic inputs to produce output spikes, they often criticize the various models' simplification that the number of synchronous EPSPs required to fire $\left(N_{\mathrm{th}}\right)$ does not depend on firing rate or previous history. Such criticism is entirely justified. In fact, our simulation could generate values of $N_{\mathrm{th}}$ varying by more than a factor of 4 , depending on such circumstances. For instance, the b.p. model required on average about 19 simulta-
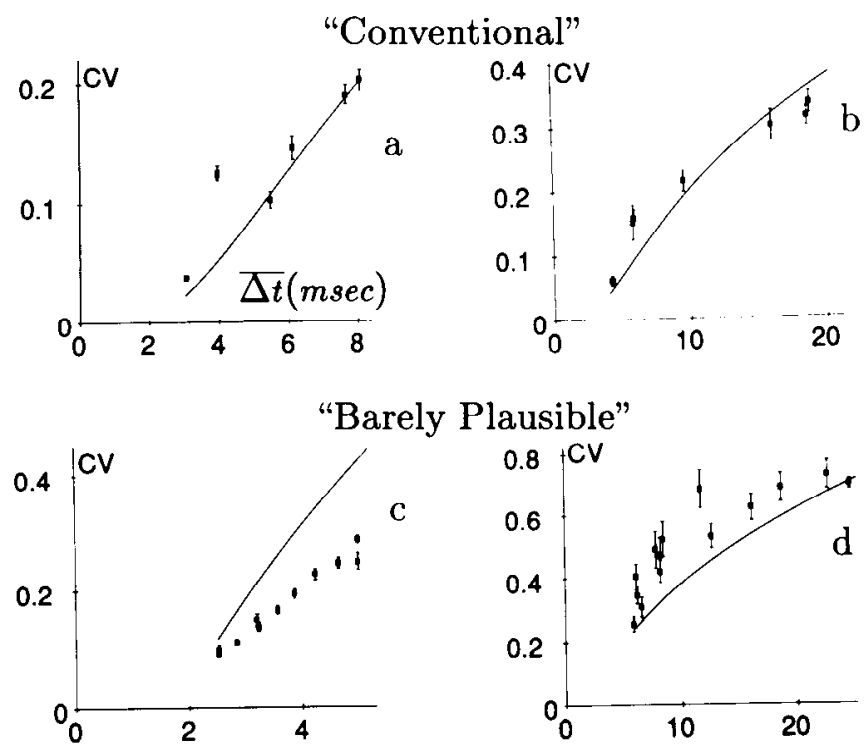

Figure 14. Comparison of $C_{1}$ from compartmental simulations with integrator models. Scattered points represent the $C_{l}$ values of simulated spike trains at various average EPSP rates; curves are predictions from the modified perfect integrator (Eq. 17), using the appropriate values of $N_{\mathrm{th}}, t_{\text {peak }}$, and initial $\overline{\Delta t}$, and with $t_{0}=1.5 \mathrm{msec}$. $C_{\mathrm{r}}$, values were calculated with $0.1 \mathrm{msec}$ bin width and 20 histograms to avoid artificially broadening the histograms. The c. model had an average EPSP rate of 400 $\mathrm{kHz}(a)$ and $103 \mathrm{kHz}(b)$; the b.p. model had an average EPSP rate of $43 \mathrm{kHz}(c)$ and $21 \mathrm{kHz}(d)$. These models required roughly $N_{\mathrm{th}}=18$ EPSPs (b.p. model) or $N_{\mathrm{th}}=130$ EPSPs (c. model) to trigger the first spike. At high firing rates the simulations produced very regular spiking, because of their refractory periods, dendritic attenuation of high-frequency signals, and nonimpulse EPSPs. At lower firing rates $(\overline{\Delta t}>5$ msec), those influences decreased, and the dominant effect became adaptation, as $I_{\mathrm{AHP}}$ increased $C_{1}$. by reducing only the DC portion of the random EPSP input current. The reasonable fits in three of the four cases suggest that the modified integrator model accounts for most of the statistical properties of the biophysical simulation (but not the monkey data), despite the model's many drastic simplifications.

neous EPSPs to fire from rest. After adaptation, a greater number was required.

To what extent could a simple analytical model capture the firing properties of the detailed pyramidal cell simulation? We chose as a model the perfect integrator with refractory period, modified for adaptation and random-height, nonimpulse EPSPs (see Eqs. 14, 16, 26, 35; we multiplied all the correction terms without considering their impact on one another). A leak term was not included, since its effect could only be studied by computer simulations; furthermore, any reasonable leak term would be overwhelmed by the adaptation term. When combined, these modifications yielded the prediction

$$
\begin{aligned}
& C_{l}=\sqrt{\frac{2}{N_{\mathrm{th}}}}\left(\frac{\overline{\Delta t} t_{0}}{\overline{\Delta t}}\right) \times \frac{1}{\left(\pi t_{\mathrm{pcak}} / \overline{\Delta t}\right)^{2}+1} \\
& \times \sqrt{\frac{\overline{\Delta t}-t_{0}}{\overline{\Delta t_{\mathrm{st}}}-t_{0}}}
\end{aligned}
$$

where $\overline{\Delta t_{s 1}}$ is the mean ISI at the start (fastest part) of the spike train. We chose $t_{0}=1.5 \mathrm{msec}$ (the minimum value in our simulations), and $t_{\text {peak }}$ directly from the corresponding simulations. For the threshold $N_{\text {th }}$ we used the number of simultaneous EPSPs 
necessary to fire the cell from rest, although other definitions could have been plausibly used instead.

Equation 17 gave a reasonable fit to three of the four simulated

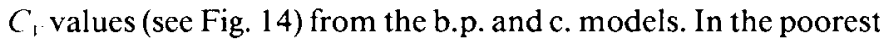
match (the fastest b.p. simulation), the variability predicted by Equation 17 was too high by a factor of $2 ; C_{1}$ values of the other two simulations were predicted within $10-20 \%$. Such good fits are surprising, because the modified integrator model includes neither dendritic effects, shunting terms, nor relative refractory period.

\section{Active dendritic simulation}

The foregoing model included voltage-dependent conductances only at the soma, leaving the entire dendritic tree passive. In that case, the neuron will act as an integrator, with low firing variability. How could dendritic nonlinearities affect our results?

It is known that dendrites in hippocampal and neocortical pyramidal cells can generate TTX-insensitive all-or-none electrical events that most likely involve calcium conductances (Wong ct al., 1979; Jones et al., 1989; Westenbrook ct al., 1990; Regehr and Tank, 1991; Amitai et al., 1992; see also Huguenard et al., 1989). But the relatively long duration of such events (20$50 \mathrm{msec}$ ) would carry little high-frequency variability in current to the soma. Because there is not much detailed data available, we conducted an explicitly unrealistic simulation of active dendritic conductances, intending only to show that they are in principle capable of producing high firing variability in response to random input.

We reasoned that the since the soma spikes in response to currents from the dendrites, we must make the dendritic currents as variable as possible. Variability in dendritic current relative to its mean sustained value can arise in general from two mechanisms: (1) fast, strong depolarizing impulses (such as spikes), which add both variable and sustained components to the dendritic current, and (2) fast repolarizing impulses, which remove the sustaincd component of current contributed by the spikes: fast repolarization increases the variable (AC) component of dendritic currents and reduces the sustained (DC) component. We chose strong values of $I_{\mathrm{DR}}$ so that the voltage at the soma returned within a few milliseconds to nearly the same voltage it had before the dendritic spike (without this very strong rectifying current, the somatic depolarization persisted, decaying slowly with the cell's passive time constant). Because the cell carried little lasting memory of a spiking event, the cell did not integrate dendritic spikes, but only fired upon the coincidence of several of them.

We therefore simulated active Hodgkin-Huxley-like conductances in the basal terminal branches, between their tip and most distal branching. We matched the mean sodium conductance to its somatic value $\left(\bar{g}_{\mathrm{Na}}=200 \mathrm{mS} \cdot \mathrm{cm}^{-2}\right)$, and used a fast potassium conductance twice that value (or 3.5 times the somatic $\bar{g}_{\mathrm{DR}}$ ) to accomplish the repolarization outlined above. Even with these very strong conductances, most neighboring dendritic terminal branches were decoupled, so that a spike in one would not necessarily fire its neighbor. In addition, most voltage-dependent conductances in the soma were removed, along with the basket cell inhibition and all apical input simulated earlier. As a result this "bare" neuron contained only Hodgkin-Huxley-like mechanisms and a passive membrane with time constant of about $30 \mathrm{msec}$. Each basal dendritic compartment was subdivided into 20 subcompartments ( 1600 subcompartments total) to ensure that the high-frequency dendritic fluc-

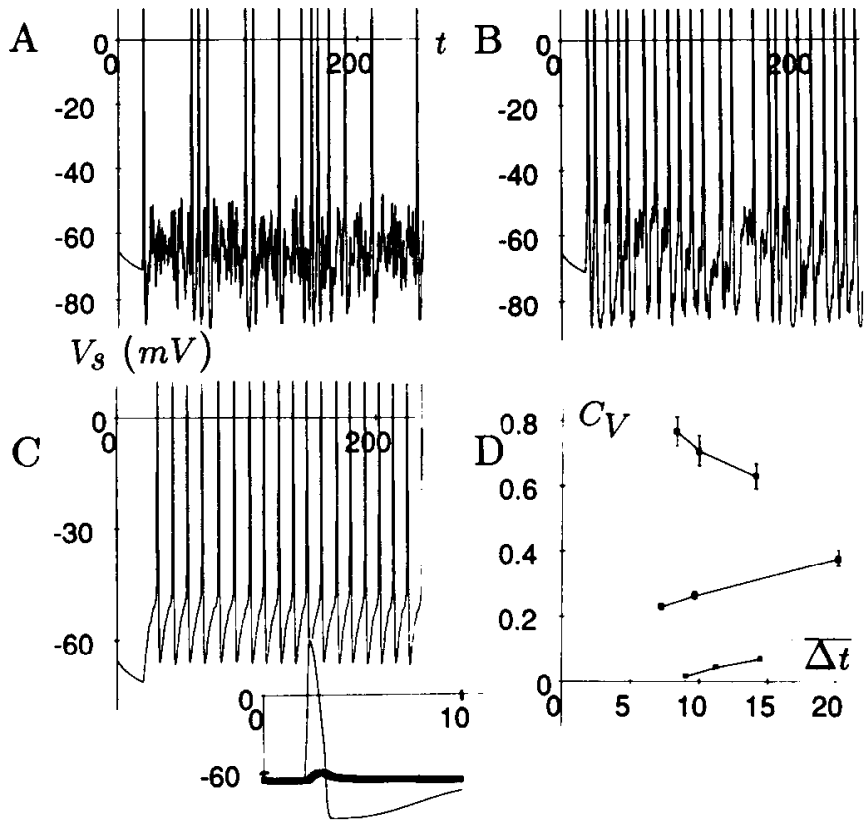

Figure 15. Highly variable spiking caused by simulated strong dendritic nonlinearities. Our layer $\mathrm{V}$ pyramidal cell described above was endowed with only Hodgkin-Huxley-like conductances at the soma and on the most distal branches of the basal dendrites; no other active currents or synaptic inhibition werc included. Dendritic spikes (thin line in inset) were triggered in each of the 42 active basal branches at a random time more than $2 \mathrm{msec}$ after its previous firing, and independent of the other branches' triggerings. $A$, The most variable somatic firing occurred for dendritic potassium conductances $g_{D R}$ twice the strength of the sodium conductance, because the strong repolarization cut short the somatic depolarization (thick line in inset), thereby preventing temporal integration. $B$, Less variable firing occurred when the dendritic $g_{\mathrm{DR}}$ was reduced to $1 / 10$ of its above value, thereby allowing spikes' depolarization to accumulate in the cell body over time and permitting temporal integration of dendritic spikes. $C$, The control case: highly regular spiking occurred in dendrites with no active conductances, as many triggering pulses were integrated to fire the cell. $D, C_{\mathrm{I}}$, values for the three aforementioned simulations: strong $I_{\mathrm{DR}}$ (top curve), weak $I_{\mathrm{DR}}$ (middle curve), and passive dendrites (bottom curve). Each squarc represents the $C_{t}$ calculated from 20 simulated trains at a constant firing rate. Only the case with strong dendritic $g_{D R}$ yielded high output firing variability consistent with the monkey data.

tuations were integrated faithfully. Apical dendrites were left passive and unstimulated because they consumed large computational resources while contributing little voltage to the soma.

Each of the 42 active dendritic terminal branches was stimulated with single triggering pulses, each pulse instantly resetting the local membrane voltage to $-40 \mathrm{mV}$ and initiating a dendritic spike. Each dendritic spike caused a peak somatic depolarization between 2 and $9 \mathrm{mV}$. Each terminal branch was stimulated independently of the others, but the input to a single terminal branch was not random: we allowed for an absolute refractory period of $2 \mathrm{msec}$ after each spike's firing before choosing a random time at which to fire it again. This requirement increased the regularity of the dendritic input and limited the speed at which the dendritic spikes could fire, hence keeping the soma's output spike rate lower than we desired.

As a result of this bombardment by dendritic spikes (up to 22 dendritic spikes/mscc), the somatic voltage fluctuated strongly about a roughly constant $-65 \mathrm{mV}$ (Fig. 15A). As desired, there was a large variability in the somatic voltage without significant sustained depolarization. Each of the three dendritic 
spike rates used produced a constant average output spike rate, so that we could analyze with a single histogram the $C_{V}$ values of each of the 20 trains simulated at that rate (Fig. 15B). $C_{\text {v }}$ values were $0.6-0.8$, significantly above those for the passivedendrite models, and in the same region as the monkey data; the variance in spike number $\sigma_{S}^{2}$ reached nearly Poisson values $(0.7 \mathrm{~S})$.

In order to isolate the relative contributions to firing variability of the dendritic sodium currents, the potassium currents, and the triggering pulses, we performed the same simulation for two related scenarios. In one scenario, we reduced $I_{\mathrm{DR}}$ currents by a factor of 10 from their values above, leaving enough delayed rectification to reset the local sodium channels but not enough to repolarize the soma significantly after a dendritic spike. This simulation naturally required less frequent dendritic spiking to fire the soma; when its output rate was adjusted to match that of the strong $-I_{\mathrm{DR}}$ case above, it produced $C_{V}$ values about half as large (0.2-0.4), suggesting that fast active rectifying currents in the dendrites can be an indispensable contribution to output variability (Fig. 15C).

A small portion of the soma's depolarization arrived not through active conductances, but merely from the triggering pulses that reset terminal branch voltages randomly to $-40 \mathrm{mV}$. We verified that the triggering pulses by themselves contributed virtually no variability to the output by eliminating all active dendritic currents, while keeping the triggering pulses; the cell's response at the same output rates above was extrememly regular $\left(C_{1}=0.02-0.07\right.$; Fig. 15D).

\section{Discussion}

We will now briefly review the assumptions underlying our spike train analysis, discuss the experiment data, and then list possible objections to our compartmental modeling efforts. We will finish by describing some of the implications of our analysis.

\section{Statistical assumption underlying our data analysis}

There exists a very rich literature concerned with the statistical analysis of spike trains using the theory of stochastic point processes (Perkel et al., 1967; Tuckwell, 1989). Almost invariably, it is assumed that the spike-generating process is a stationary one, so that the underlying probability distribution of these point events does not change with time, or depend on a "starting" time (Burns and Webb, 1976; Correia and Landolt, 1977; Teich et al., 1977; Lansky and Radil, 1987). However, the spike trains used in our study all occur following stimulation, and their response is nonstationary. The most prominent such nonstationarity is the decrease in firing rate with time (Fig. 1C,D), reflecting both adaption processes intrinsic to the cell as well as network effects.

Since we were not concerned with the detailed fitting of analytical distribution functions to the ISI histograms of these spike trains, we tried to account for the nonstationarity inherent in the data by using the simple normalization process described in the Parameters and Normalization Procedure section. That method calculated an approximate instantaneous firing rate from the PSTH and the stimulus efficacy, and used that rate to create several separate, near-stationary histograms for $C_{V}$ analysis.

We also concluded that most of the firing variability arises at a fast time scale, that is, milliseconds, rather than at the slower time scale of varying average rates, that is, tens of milliseconds. In a different study, we had computed the autocorrelation functions associated with the single-cell data derived from macaque area MT (W. Bair, C. Koch, W. Newsome, and K. Britten, unpublished observations). The absence of a broad central peak around the origin-associated with a process whose mean rate varicd slowly over timc (scc Fig. 7 in Pcrkel ct al., 1967)suggested that adjacent fast ISIs were uncorrelated, so that a fluctuating mean rate did not contribute to the high firing variability we observed.

But in some MT cells a broad autocorrelogram peak (50-200 msec) did exist in the absence of any structure in the PSTH; we interpret this to mean that the firing rate fluctuated randomly. To estimate the fast-time-scale variability in that situation, we computed $C_{V}$ from many tiny histograms of only ten adjacent ISI's each. Those $C_{V}$ values were widely scattered about a mean $10 \%-20 \%$ below the $C_{V}$ computed by the multi-histogram method (see Parameters and Normalization Procedure section), suggesting that those neurons fire quite irregularly at fast as well as slow time-scales.

\section{The variability of cortical cell firing}

We measured the degree of variability of the neuronal spike discharge in a large number of nonbursting striate and extrastriate cortical cells in two different but related manners. One study was of the variability in the intervals between consecutive action potentials. Its principal result is shown in Figure 3: for firing rates up to several hundred Hertz, the value of $C_{V}$ is close to 1 (characteristic of a random Poisson process) for both $\mathrm{Vl}$ and MT cells.

We also measured the variability in the number of action potentials in a single train for both sets of data (Fig. 5). Our finding that the variance of cell firing increases roughly linearly with the mean response rate is well known for cells in cat and monkey primary visual cortex (Heggelund and Albus, 1978; Tolhurst et al., 1983; Parker and Hawken, 1985; Vogels et al., 1989; Zohary et al., 1990), and has recently also been established for cells in area MT of the alert macaque monkey (Snowden et al., 1992). Our results here are compatible with the known literature, and are also approximately consistent with a description of spiking as a Poisson process (see above).

\section{Analytical results}

In an attempt to understand the origin of the observed variability in the neuronal discharge, we analyze the variability of the impulse activity of different integrate-and-fire models. Our primary assumption is that these models - as well as the passive compartmental models - spatially and temporally integrate synaptic input from a large number of independent processes. Our results can be qualitatively explained by the Central Limit Theorem, which states that as the number $n$ of incoming independent random variables $x_{i}$ goes to infinity, the random variable defined by the mean over $x_{i}$, that is, $\bar{x}=(1 / n) \Sigma_{i=1}^{n} x_{i}$, has an asymptotically normal (i.e., Gaussian) distribution, with mean identical to the mean of the population $x_{i}$ and with SD scaling as $1 \sqrt{n}$ of the population's SD. In other words, if a neuron can only be brought to fire action potentials by summing over dozens or more of independent synaptic inputs, it should fire very regularly!

This intuition is born out by an analysis of the $C_{V}$ of different integrator models, which are summarized in the contour plot (Fig. 8). The high $C_{v}$ value we observe experimentally can only be obtained by either assuming that $N_{\text {th }}$ is very small, that is, that one to two inputs are sufficient to trigger the cell (Fig. 7), or that the time constant $\tau$ is a fraction of a millisecond, thereby 
preventing any effective temporal integration from occurring. Further modifications to the leaky-integrator model (see Appendix), such as hyperpolarizing (adapting) currents and the finite width and variable amplitude of EPSPs, do not change in any significant manner our fundamental conclusion that integrator models produce very regular output trains at high firing rates.

\section{Biophysical detailed simulations}

It can be argued that simple integrator models do not provide a realistic description of cortical pyramidal cells. In order to satisfy ourselves that a passive-dendrite neuron that integrates many independent EPSPs is inconsistent with the measured high variability, we numerically simulated the dynamical properties of an HRP-injected and reconstructed neocortical, layer V, pyramidal cell. While the morphology and electrophysiology of this cell were derived from cat visual cortex, its properties are not likely to differ fundamentally from those in monkey visual cortex, the source of our variability data. We acknowledge that some of our monkey cells - especially the fastest-firing onesmay have been rapidly firing interneurons rather than pyramidal cells (Agmon and Connors, 1992). But we chose a pyramidal (rather than interneuron) cell model because pyramidal cells are far more common and larger, and hence probably represent the majority of the monkey cells recorded.

Conceptually, we would like to distinguish these simulations according to whether the cell acts as an integrator or whether it acts as a high-fidelity temporal coincidence detector. Accordingly, for the majority of our simulations, we assumed that the dendritic tree contained no voltage-dependent membrane conductances (integrator mode), while in a second, more exploratory set of simulations we endowed the distal part of the basal dendritic tree with strong nonlinearities (coincidence mode).

\section{Passive dendrites: integrator mode}

If the simulated pyramidal cell was bombarded by massive amounts of fast, excitatory synaptic conductance inputs of the non-NMDA type, such that it fired at the high firing rates observed in our monkey data, then its output firing-even in the b.p. simulation - was much more regular than the monkey cells' firing. Both the experimental and the modeling data are compared by the variability in the number of action potentials per trial, and by the rate-normalized $C_{V}$. Thus, in spite of the complex dynamics of the seven voltage- and time-dependent currents at the soma, the simulated cell essentially still acted like an integrator, and its low variability was predicted by a modified integrator model (Eq. 17; Fig. 14).

Similar to the integrate-and-fire model discussed above, high $C_{\text {I }}$ values could only be obtained if single EPSPs were very large (greater than $10 \mathrm{mV}$, thereby reducing $N_{\text {th }}$ to 1 or 2 ), or if the passive time constant was in the submillisecond range. But these ranges are excluded by intracellular recordings. Evidence from neocortical and hippocampal slice recordings report a range of unitary EPSPs between $0.05 \mathrm{mV}$ and $3 \mathrm{mV}$, with the majority of averaged EPSPs less than $0.5 \mathrm{mV}$ (McNaughton et al., 1981; Thompson et al., 1988; Sayer et al., 1990; Mason et al., 1991). I hose measurements do include the multiple boutons that single axons often make on individual cells. Occasionally, much larger EPSPs have becn observed (C. Stevens, personal communication). And recordings from the cell body of coritcal pyramidal cells yield values of $\tau$ on the order of $10-20 \mathrm{msec}$ in the intact animal (Creutzfeldt et al., 1974; Douglas et al., 1991), and much larger values in slice neurons using the patch-clamp technique (Spruston and Johnson, 1991). We did not simulate any voltagedependent (NMDA) synaptic input, since the long decay times (20-50 msec; Hestrin et al., 1990) of the NMDA-associated conductance would dramatically reduce variability in synaptic currents. Modification of other cellular parameters, such as lowering the threshold for initiation of action potentials or blocking all but the fast sodium and the delayed-rectifier potassium current, had very little effect on the $C_{v}$ values.

While an inherently random firing mechanism can account for the high variability we observe in monkey cells, some research (in other neuron types) has suggested that the spike-firing mechanism is inherently very reliable. Calvin and Stevens (1968) concluded that cat spinal motoneurons derive at least $90 \%$ of their already small firing-time variability from variability in synaptic currents. Bryant and Segundo (1976) found that various neurons in Aplysia gave virtually identical response patterns to repeated injections of white-noise current. This reliability occurs because the types of statistical fluctuation expected from spikegenerating mechanisms - for instance, random channel openings-have small quantal size, so their collective effects are reasonably constant over a reasonably large membrane area (Strassberg and DeFelice, in press). In general, only the largest quantal effects - such as EPSP arrivals - will contribute significantly to firing variability.

\section{Active dendrites: coincidence mode}

We also simulated active dendritic conductances whose random triggering maximized the cell's firing variability. We found it very difficult to "construct" a pyramidal cell that fires as irregularly as the monkey cells. For such events to cause highly variably somatic firing, the dendritic spikes must be large, fast, and strongly repolarizing. Only under these conditions do we see high variability for spikes before adaptation sets in. Yet we do not claim that such dendritic nonlinearities exist, but only that they can, in principle, explain the observed variability.

The most important characteristic of this simulation, and the reason why it produced such high variability of output firing, was that it did not perform temporal integration of dendritic spikes, but only coincidence detection among them. This property became evident in the cell's strikingly strong response to slightly synchronized inputs: when dendritic spikes were reorganized to fire in simultaneous pairs (rather than singly) at the same average rate as before, the cell's output firing rate increased by over $50 \%$. Such coincidence detection is analogous to the "logic operations" postulated to take place among dendritic spines (Shepherd et al., 1989) and has been discussed for leakyintegrator models operating at much slower rates (Bugmann, 1991). But in such a scheme, the individual output spikes would represent the fundamental elements of logical computations at the millisecond scale, rather than mere "noise" in an average firing rate that is averaged out over tens to hundreds of milliseconds.

\section{Network effects}

Of the many parameters we need to reevaluate in light of this discrepency, perhaps the most intriguing is the possibility that the individual synaptic events impinging onto a cortical neuron are not independent after all. In that case, the Central Limit Theorem would not apply any more. In particular, what if the EPSPs arriving from different neurons were synchronized? What 
degree of synchrony could account for the firing variability of our cells, and where might it come from?

Weakly synchronized EPSPs would not be sufficient. In all of the foregoing models (except the $\tau<1 \mathrm{msec}$ case), the neuron's output variability directly reflects the variability of its synaptic input current. Therefore, if many small FPSPs are to account for the observed high output variability, they must be strongly synchronized, so that the resulting current is just as variable as a current composed of individual $10-15 \mathrm{mV}$ events (i.e., the effective $N_{\text {th }} \leq 2$ ). Any significant number of non-synchronized EPSCs would create a nearly DC "background" current, which would reduce the variability of the net input current and hence of the output firing. Such synchrony might result from network effects such as burst synchronization (Bush and Douglas, 1991; Koch and Schuster, 1992).

For synchronized firing to explain the $C_{V}$ results presented here, a majority of the EPSPs must be coincident at the millisecond scale. Highly synchronized EPSPs were first proposed as the "reverberation" in a "cell assembly" by Hebb (1949), and later as "synfire chains" by Abeles (1990). As cvidence, Abeles cites millisecond precision in repeated interspike intervals observed in various locations of monkey cortex in his laboratory (Abeles, 1982). Similarly precise ISIs are reported by Strehler and Lestienne (1986) for monkey visual cortex, Frostig et al. (1985) for cat medial frontal cortex, and Legendy and Salcman (1985) for cat striate cortex. But highly synchronized inputs would raise serious questions about the "stochastic" nature of neurons (Knight, 1972; Sejnowski, 1981; Hinton and Sejnowski, 1986), and the resulting justification for population coding and massive redundancy.

\section{Conclusion}

According to our current understanding of pyramidal cells, only a few situations could cause near-random, fast firing in these cells: a very strong inhibitory leak (leading to an effective membrane time constant $\tau=0.2 \mathrm{msec}$ ); extremely strong synaptic events ( $>10 \mathrm{mV}$ depolarization per EPSP); strong and fast nonlinear dendritic all-or-none events, with fast repolarization; or highly synchronized, nonrandom synaptic input. In short, either the cell must have extremely large, fast depolarizations, or it must have a very fast mechanism for repolarizing the membrane during "integration." In both these cases, the high ISI variability results directly from an equally high variability in the currents arriving at the soma. Neither case corresponds to temporal integration over a large number of small, independent, excitatory synaptic events.

The traditional view of cortical firing variability has been that information is only carried in the average spike rate (frequency code); scatter about that rate represents random "noise," whose particular structure is of no use. According to this view, a neuron that fires very randomly carries uncertain information, because of the inevitable scatter in the number of counts accumulated during integration-only a few distinct counting rates can be distinguished in a short integration time. Thus, a highly irregular neuron is the "worst possible" at carrying information in its average rate. Stein (1967b) found that such a frequency-coding neuron has a channel capacity decreasing roughly as $\log \left(1 / C_{V}\right)$ for large integration times. While such frequency coding is very inefficient, it is robust to perturbation of individual spike times, and it does not require complicated postsynaptic neurons to "decode" its message. Furthermore, high variability may have useful properties. It can help a neuron to "explore" its nearby synaptic vector space during unsupervised learning (Mazzoni et al., 1991). And it may enable neurons to implement multiplicative (quadratic) computations (Srinivasan and Bernard, 1976; Suarez and Koch, 1989; Koch and Poggio, 1992).

The alternative view is that each spike's arrival time signifies an independent message of some sort (an asynchronous binary pulse code). If each message (spike) has the same probability of arrival, independent of the other messages, then the resulting spike train is Poisson (by definition), and the spike train carries the maximum amount of Shannon information possible for its fixed bandwidth and firing rate (Stein, 1967b; the less predictible a spike is, the more information it carries). Thus, a highly irregular neuron would be the "best possible" for carrying information in its individual spike times, although the nature of the information encoded and the ability of subsequent neurons to use it may be unclear. Further experimental and computational studies are required to determine whether cortical computations occur at the millisecond level.

\section{Appendix: Modifications to the Perfect Integrator Model}

EPSC width

The integrate-and-fire neuron model assumes that EPSPs result from instantaneous current impulses, which carry equal Fourier components at all frequencies and hence produce a frequencyindependent input to the neuron. But in fact an actual EPSC is not a delta function, but arises from a conductance approximately of the form

$$
g_{s}(t) \propto t \exp \left(-t / t_{\text {peak }}\right) .
$$

As long as the membrane potential is well below the synapse's reversal potential, we can approximate the synaptic current by

$$
I_{s}(t) \propto t \exp \left(-t / t_{\text {peak }}\right)
$$

The nonzero width of these EPSCs blurs a pulse train's highfrequency information. We want to find the amount of that blurring in synaptic current at some test frequency $f$; the blurring is given by the decrease in Fourier amplitude of the blurred current train at $f$ relative to the unblurred spike-like train.

A train of realistic current impulses is given by convolving the individual synaptic current $I(t)$ with the random "comb function" $\Sigma_{i} \delta\left(t-t_{i}\right)$ of the spike-like inputs,

$$
I(t)=\sum_{i} \delta\left(t-t_{i}\right) * I_{s}(t)
$$

The Fourier amplitude of $I(t)$ at frequency $f$ is just the product of the separate Fourier amplitudes $\mathcal{F}_{f}\left(I_{s}(t)\right)$ and $\mathcal{F}_{f}\left(\delta\left(t-t_{i}\right)\right.$ ) (by the Fourier convolution theorem). The random comb contribution to $C_{1}$. has already been determined (Eq. 12), so we only need to examine synaptic current smoothing by the single-event term $\mathcal{F}_{f}\left(I_{s}(t)\right)$. That attenuation $A(f)$ of a single EPSC relative to a delta function is the EPSC's Fourier amplitude at $f$, normalized by its area (found by using $f=0$ );

$$
\begin{aligned}
& A(f)=\frac{\left|\mathcal{F}_{f}\left(I_{s}(t)\right)\right|}{\left|\mathcal{F}_{0}\left(I_{s}(t)\right)\right|}, \\
& A(f)=\frac{\left|\int_{-\infty}^{\infty} I_{s}(t) \exp (2 \pi i f t) d t\right|}{\int_{-\infty}^{\infty} I_{s}(t) d t}, \\
& A(f)=\frac{1}{\left(2 \pi f t_{\text {peak }}\right)^{2}+1} .
\end{aligned}
$$


At what frequency $f$ do we wish to evaluate this attenuation? Suppose that some spikes occur with mean interval $\overline{\Delta t}$ and some scatter $\epsilon$ about that mean, so that sequential intervals are

$$
\left\{\Delta t_{i}\right\}=(\Delta t+\epsilon),(\Delta t-\epsilon),(\Delta t+\epsilon),(\Delta t-\epsilon), \ldots
$$

This simplified example, with variability present only at a single frequency, has periodicity $2 \overline{\Delta t}$, so that

$$
f=1 / 2 \overline{\Delta t}
$$

(this is identical to the result from the Nyquist Sampling Theorem). Using this estimate of $f$, we conclude that

$$
\begin{aligned}
& A(f) \approx 1 /\left[\left(\pi t_{\text {prak }} / \overline{\Delta t}\right)^{2}+1\right], \\
& A(f) \approx 0.5,
\end{aligned}
$$

for $\overline{\Delta t}=4 \mathrm{msec}$ and $t_{\text {peak }}=1.5 \mathrm{msec} . A(f)$ represents the attenuation of current variability at $f$ reaching an integrate-and-fire neuron, due to synaptic blurring. If we suppose that this attenuation of current roughly corresponds to the attenuation of $C_{1}$. (see Eq. 28), then $A(f)$ (Eq. 26) should be multiplied by the perfect-integrator prediction (Eq. 12). This blurring makes it morc difficult to reconcile the observed variability with theory, even at low $N_{1 \mathrm{~h}}$ : how can a neuron produce output varations whose frequency is higher than that contained in a single EPSP?

\section{Adaptation}

The spike rate in the monkey cells decreases by about half in the first $100-300 \mathrm{msec}$ of a train. Some of this decrease is likely due to a decrease in synaptic input to the cell, but a major contribution to this slowdown is probably due to the "spike adaptation" potassium currents. How will these outward currents affect the predicted $C_{l}$, values if the synaptic input remains unchanged?

Because $I_{\text {AHP }}$ (considered as a single current) has a reversal potential much lower than the resting potential, it can be modeled (to first order) as a outward sustained current, which partly cancels the inward sustained portion of the EPSC while leaving its fluctuations unchanged. As a result, the mean ISI will increase during this adaptation. We wish to approximate the influence of this increased ISI on the perfect integrator with absolute refractory period (Refractory Period section).

Let us assume a low variability in the perfect-integrator model $\left(C_{1}: \ll 1\right)$, so that the proportional variation in time $C_{1}=\sigma_{\Delta l} /$ $\overline{\Delta t}$ to reach a fixed threshold of $N_{\mathrm{th}}$ is roughly the same as the proportional variation in the rclative synaptic depolarization $\delta V / V_{\mathrm{th}}$ arriving in the mean time interval $\overline{\Delta t}$ :

$$
\begin{aligned}
\frac{\sigma_{\Delta t}}{\overline{\Delta t}} & \approx \frac{\delta V}{V_{\mathrm{th}}}, \\
C_{\mathrm{IAHP}_{\mathrm{P}}} & \approx \delta V_{\mathrm{AHP}} / V_{\mathrm{AHP}} .
\end{aligned}
$$

As the outward current increases, firing frequency decreases, and the mean ISI during adaptation $\overline{\Delta t}_{\mathrm{AHP}}$ will increase above the ISI at the start of the train $\overline{\Delta t}_{\mathrm{st}}$,

$$
\overline{\Delta t}_{\mathrm{AHP}}>\overline{\Delta t}_{\mathrm{st}} \text {. }
$$

But by assumption the depolarization necessary to reach firing threshold will remain constant:

$$
V_{\mathrm{AHP}}=V_{\mathrm{st}}=V_{\mathrm{th}} \text {. }
$$

How much variation in depolarization $\left(\delta V_{\mathrm{AHP}}\right)$ will accumulate during that longer ISI? The rate of random EPSCs is assumed to be unchanged by the addition of outward adaptation current. So it is clear that the expected number of EPSCs arriving in $\overline{\Delta t}_{\mathrm{AHP}}$ will increase, in proportion to $\overline{\Delta t}_{\mathrm{AHP}}$. But the variation about that mean will not increase linearly with interval duration, but rather as its square root, as occurs in accumulating any large number of independent events in a single time period:

$$
\delta V_{\mathrm{AHP}}^{\prime} \approx \delta V_{\mathrm{st}} \sqrt{\frac{\overline{\Delta t}_{\mathrm{AHP}}}{\Delta t_{\mathrm{st}}}} .
$$

The above formula applies only during the integration period, and is thus only valid for the perfect integrator without refractory period. Recognizing that the observed ISI contains the refractory period $t_{0}$ means that the true integration time is $\overline{\Delta t}-t_{0}$

$$
\delta V_{\mathrm{AHP}} \approx \delta V_{\mathrm{st}} \sqrt{\frac{\overline{\Delta t}_{\mathrm{AHP}}-t_{0}}{\overline{\Delta t_{\mathrm{st}}-t_{0}}}} .
$$

Combining Equations 29, 31, and 33 gives us an expression for $C_{r}$ as a function of ISI for an adapting spike train:

$$
\begin{aligned}
& C_{V_{\mathrm{AHP}}} \approx\left(\frac{\delta V_{\mathrm{st}}}{V_{\mathrm{th}}^{\prime}}\right) \sqrt{\frac{\overline{\Delta t}_{\mathrm{AHP}}-t_{0}}{\overline{\Delta t_{\mathrm{st}}}-t_{0}}},
\end{aligned}
$$

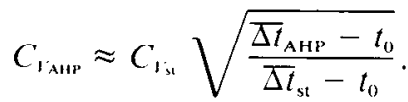

This rough result indicates that during spike adaptation, $C_{I}$, will rise with the square root of $\overline{\Delta t}$, much faster than the leakyintegrator model suggests. Such a sharp increase in variability is indeed observed both in simulations (Figs. 10-14) and in the monkey data. But the magnitude of the rise in $C_{I}$, in simulations is not sufficient to account for the larger discrepency between theoretical and observed $C_{1}$, nor can it account for the high $C_{\text {, }}$ observed for early spikes, before the onset of adaptation.

\section{References}

Abeles M (1982) Local cortical circuits. Berlin: Springer.

Abeles M (1990) Corticonics. Cambridge: Cambridge UP.

Aertsen A, Gerstein G, Habib M, Palm G (1989) Dynamics of neuronal firing correlation: modulation of 'effective connectivity.' J Neurophysiol 61:900-917.

Agmon A, Connors B (1992) Correlation between intrinsic firing patterns and thalamocortical responses of neurons in mouse barrel cortex. J Neurosci 12:319-329.

Amitai Y, Friedman A, Connors B, Gutnick M (1992) Regenerative electrical activity in apical dendrites of pyramidal cells in neocortex. $\mathrm{J}$ Neurophysiol, in press.

Bernander Ö, Douglas R, Martin K, Koch C (1991) Synaptic background activity determines spatio-temporal integration in single pyramidal cells. Proc Natl Acad Sci USA 88:1569-1573.

Britten KH, Shadlen MN, Newsome WT, Movshon A (1992) The analysis of visual motion: a comparison of neuronal and psychophysical performance. J Neurosci 12:4745-4765.

Bryant H, Segundo J (1976) Spike initiation by transmembrane current: a white-noise analysis. J Physiol (Lond) 260:279-314.

Bugmann G (1990) Irregularity of natural spike trains simulated by an integrate-and-fire neuron. In: Extended abstracts, 3rd International Symposium on Bioelectronic and Molecular Electronic Devices, pp 105-106. Toranomon, Japan: FED.

Bugmann G (1991) Neural information carried by one spike. In: Proceedings of the $2 \mathrm{~d}$ Australian Conference on Neural Networks (ACNN'91) (Leang P, Jabri M, eds), pp 235-238. Sydney: Sydney UP.

Burns BD, Webb AC (1976) The spontaneous activity of neurones in the cat's visual cortex. Proc R Soc Lond [Biol] 194:211-223. 
Bush R, Douglas P (1991) Synchronization of bursting action potential discharge in a model network of neocortical neurons. Neural Comput 3:19-30.

Calvin W, Stevens C (1968) Synaptic noise and other sources of randomness in motoneuron interspike intervals. J Neurophysiol 31:574587.

Cinlar E (1972) Superposition of point processes. In: Stochastic point processes (Lewis PAW, ed), pp 549-606. New York: Wiley.

Correia MJ, Landolt JP (1977) A point process analysis of the spontaneous activity of anterior semicircular canal units in the anesthetized pegeon. Biol Cybern 27:199-213.

Creutzfeld O, Lux H, Nacimiento A (1964) Intrazelluläre Reizung corticalen Nervenzellen. Pflügers Arch 281:129-151.

Douglas R, Martin K (1991) A functional microcircuit for cat visual cortex. J Physiol (Lond) 440:735-769.

Douglas R, Martin K, Whitteridge D (1991) An intracelluar analysis of the visual responses of neurones in cat visual cortex. J Physiol (Lond) 440:659-696.

Frostig RD, Frostig Z, Frysinger RC, Schechtman VL (1985) Multineuron analysis reveals complex patterns of interaction among neurons in forebrain networks and cardiorespiratory parameters during sleep-waking states. Soc Neurosci Abstr 11:1020.

Hebb DO (1949) The organization of behavior. New York: Wiley.

Heggelund P, Albus K (1978) Response variability and orientation in single cells in striate cortex of cat. Exp Brain Res 32:197-211.

Hestrin S, Sah P, Nicoll R (1990) Mechanisms generating the time course of dual component excitatory synaptic currents recorded in hippocampal slices. Neuron 5:247-253.

Hines M (1989) A program for simulation of nerve equations with branching geometries. Int $\mathrm{J}$ Biomed Comput 24:55-68.

Hinton G, Sejnowski T (1986) Learning and relearning in Boltzmann machines. In: Parallel distributed processing, Vol 1 (Rumelhart D, McClelland J, eds), pp 282-317. Cambridge, MA: MIT Press.

Huguenard J, Hamill O, Prince D (1989) Sodium channels in dendrites of rat cortical pyramidal neurons. Proc Natl Acad Sci USA 86:24732477.

Jones OW, Kunze DJ, Angelides KJ (1989) Localization and mobility of $\Omega$-cytotoxin sensitive $C \mathrm{a}^{++}$channels in hippocampal CAl neurons. Science 244:1189-1191.

Knierim J, Van Essen D (1992) Neuronal responses to static textural patterns in area $\mathrm{V} 1$ of the alert macaque monkey. J Neurophysiol 67 : $961-980$.

Knight B (1972) Dynamics of encoding in a population of neurons. $J$ Gen Physiol 59:734-766.

Koch C, Poggio T (1992) Multiplying with synapses. In: Single neuron computation (McKenna T, Davis J, Zornetzer S, eds), pp 315-345. New York: Academic.

Koch C, Schuster H (1992) A simple network showing burst synchronization without frequency-locking. Neural Comput 4:211-223.

Komatsu Y, Nakajima S, Toyama K, Fetz E (1988) Intracortical connectivity revealed by spike-triggered averaging in slice preparations of cat visual cortex. Brain Res 442:359-362.

Lansky P, Radil T (1987) Statistical inference on spontaneous neuronal discharge patterns. Biol Cybern 55:299-311.

Lansky P, Smith C (1989) The effect of a random initial value in neural first-passage-time models. Math Biosci 93:191-215.

Legendy C, Salcman M (1985) Bursts and recurrences of bursts in the spike trains of spontaneously active striate cortex neurons. J Neurophysiol 53:926-939.

Mason A, Nicoli A, Stratford K (1991) Synaptic transmission between individual pyramidal neurons of the rat visual cortex in vitro. $\mathrm{J} \mathrm{Neu}$ rosci 11:72-84.

Mazzoni P, Andersen R, Jordan M (1991) A more biologically plausible learning rule for neural networks. Proc Natl Acad Sci USA 88 : 4433-4437.

McClurkin J, Optican I, Richmond B, Gawne T (1991) Concurrent processing and complexity of temporally encoded neuronal messages in visual perception. Science 253:675-677.

McNaughton B, Barnes C, Andersen P (1981) Synaptic efficacy and EPSP summation in granule cells of rat fascia denta studied in vitro. J Neurophysiol 46:952-966.

Mikami A, Newsome W, Wurtz R (1986) Motion selectivity in macaque visual cortex: I. Mechanisms of direction and speed selectivity in extrastriate area MT. J Neurophyiol 55:1308-1327.

Newsome W, Pare E (1988) A selective impairment of motion per- ception following lesions of the middle temporal visual area (MT). J Neurosci 8:2201-2211.

Newsome W, Britten K, Movshon JA, Shadlen M (1989a) Single neurons and the perception of motion. In: Neural mechnanisms of visual perception (Man-Kit Lam D, Gilbert C, eds), pp 171-198. The Woodlands, TX: Portfolio.

Newsome W, Britten K, Movshon JA (1989b) Neural correlates of a perceptual decision. Nature 341:52-54.

Noda H, Adey R (1970) Firing variability in cat association cortex during sleep and wakefulness. Brain Res 18:513-526.

Parker A, Hawken M (1985) Capabilities of monkey coritcal cells in spatial-resolution tasks. J Opt Soc Am 2:1101-1114.

Perkel DH, Gerstein G, Moore G (1967) Neuronal spike trains and stochastic point processes. Biophys J 7:391-418.

Poggio T, Viernstein L (1964) Time series analysis of impulse sequences of thalamic somatic sensory neurons. J Neurophysiol 27 517-545.

Regehr WG, Tank DW (1991) Postsynaptic NMDA receptor-mediated calcium accumulation in hippocampal CAI pyramidal cell dendrites. Nature 345:807-810.

Sayer R, Friedlander M, Redman S (1990) The time course and amplitude of EPSPs evoked at synapses between pairs of CA3/CA1 neurons in the hippocampal slice. J Neurosci 10:826-836.

Sejnowski T (1981) Skeleton filters in the brain. In: Parallel models of associative memory (Hinton G, Anderson J, eds), pp 189-208. Hillsdale, NJ: Erlbaum.

Shepherd G, Woolf T, Carnevale N (1989) Comparisons between active properties of distal dendritic branches and spines: implications for neuronal computations. J Cogn Neurosci 1:273-286.

Snowden RJ, Treue S, Andersen RA (1992) The response of neuron in areas V1 and MT of the alert rhesus monkey to moving random dot patterns. Exp Brain Res 88:389-400.

Softky W, Koch C (1992) Cortical cells should fire regularly, but do not. Neural Comput 4:643-646.

Spruston N, Johnson D (1991) Perforated patch-clamp analysis of the passive membrane properties of three classes of hippocampal neurons. J Neurophysiol 67:508-529.

Srinivasan M. Bernard G (1976) A proposed mechanism for multiplication of neural signals. Biol Cybern 21:227-236.

Stein R (1967a) Some models of neuronal variability. Biophys J 7: $37-68$.

Stein R (1967b) The information capacity of nerve cells using a frequency code. Biophys J 7:797-826.

Strassberg A, DeFelice L (in press) Limitations of the Hodgkin-Huxley formalism. Neural Comput, in press.

Strehler B, Lestienne R (1986) Evidence on precise time-coded symbols and memory of patterns in monkey cortical neuronal spike trains. Proc Natl Acad Sci USA 83:9812-9816.

Suarez H, Koch C (1989) Linking linear threshold units with quadratic models of motion perception. Neural Comput 1:318-320.

Teich M, Matin L, Cantor B (1978) Refractoriness in the maintained discharge of the cat's retinal ganglion cell. J Opt Soc Am 68:386-401.

Thompson A, Girdlestone D, West D (1988) Voltage-dependent currents prolong single-axon postsynaptic potentials in layer III pyramidal neurons in rat neocortical slices. J Neurophysiol 60:1896-1907.

Tolhurst D, Movshon J, Dean A (1983) The statistical reliability of signals in single neurons in cat and monkey visual cortex. Vision Res 23:775-785.

Tuckwell HC (1989) Stochastic processes in the neurosciences. Philadelphia: Society for Industrial and Applied Mathematics.

Vogels R, Spileers W, Orban GA (1989) The response variability of striate cortical neurons in the behaving monkey. Exp Brain Res 77: 432-436.

Westenbrook R, Ahlijinian MK, Catterall WA (1990) Clustering of L-type $\mathrm{Ca}^{2+}$ channels at the base of major dendrites in hippocampal pyramidal neurons. Nature 347:281-284.

Wilbur WJ, Rinzel J (1983) A theoretical basis for large coefficient of variation and bimodality in neuronal interspike interval distributions. J Theor Biol 105:345-368.

Wong R, Prince D, Basbaum A (1979) Intradendritic recordings from hippocampal pyramidal neurons. Proc Natl Acad Sci USA 76:986990.

Zohary E, Hillman P, Hochstein S (1990) Time course of perceptual discrimination and single neuron reliability. Biol Cybern 62:475-486. 